\title{
Extensional deformation, stress relaxation and necking failure of viscoelastic filaments ${ }^{1}$
}

\author{
Minwu Yao ${ }^{\mathrm{a}}$, Gareth H. McKinley ${ }^{\mathrm{b}, *}$, Benoit Debbaut ${ }^{\mathrm{c}}$ \\ a Ohio Aerospace Institute, 22800 Cedar Point Road, Brook Park, OH 44142, USA \\ ${ }^{\mathrm{b}}$ Dept. of Mechanical Engineering, Massachusetts Institute of Technology, Cambridge, MA 02139, USA \\ ' Polyflow, 16 Place de 1'Université, B-1348 Louvain-la-Neuve, Belgium
}

Received 14 April 1998; revised 15 May 1998

\begin{abstract}
We investigate the transient viscoelastic behavior of weakly strain-hardening fluids in filament stretching devices during uniaxial elongation and following the cessation of stretching. The numerical results are compared with experimental observations on a concentrated shear-thinning polystyrene solution which is well characterized by a multi-mode Giesekus model. The finite element computations incorporate the effects of viscoelasticity, surface tension, and fluid inertia and the time-dependent moving-boundary problem is solved using the code POLYFLOW. A detailed comparison of multi-mode computations with single-mode solution is presented in order to examine the differences in the predicted viscoelastic behavior and the role of the fluid relaxation spectrum. The evolution in the transient Trouton ratio at different deformation rates is compared with experimental measurements and with the theoretical predictions of ideal homogeneous uniaxial elongation. Simulations of the filament stretching device using the multi-mode viscoelastic model demonstrate a significant improvement in the agreement between the predicted and observed extensional viscosity at short times. The computed Trouton ratio is also in good agreement with theoretical expectations for ideal homogeneous uniaxial extension, despite the strongly nonhomogeneous viscoelastic necking of the fluid column observed during elongation in the filament stretching device. Following the cessation of elongation, numerical simulations predict an interesting and complex evolution in the kinematics of the fluid filament. Initially the tensile stresses in the column relax in the non-linear form predicted theoretically, indicating that filament stretching devices can be used to monitor transient extensional stress relaxation, provided that the evolution of the tensile force at the end-plate and the filament radius at the mid-plane are carefully measured. However, at longer times after cessation of stretching, the local extension rate at the axial mid-plane begins to increase rapidly, leading to a 'necking failure' that is greatly accelerated compared to that expected in a corresponding Newtonian filament. The calculations show that this unstable necking is not driven solely by the surface tension but also by the viscoelasticity of the fluid, and is coupled with significant elastic recoil of the material near the end-plates. The rate of necking in the column is a sensitive function of the extensional viscosity predicted by the constitutive model, in particular the magnitude and the rate of strain-hardening that occurs during uniaxial elongation. This phenomenon can also be simply and accurately described by an appropriate set of coupled onedimensional thin filament equations that use the finite element computations to provide a suitable initial condition for the axial distribution of the polymeric stresses in the filament. (C) 1998 Elsevier Science B.V. All rights reserved.
\end{abstract}

\footnotetext{
* Corresponding author. Tel.: +1-617-258-0754; fax: +1-617-258-8559; e-mail: gareth@ mit.edu

${ }^{1}$ Dedicated to Professor Marcel J. Crochet on the occasion of his 60th birthday.
} 
Keywords: Extensional flow; Stress relaxation; Fluid dynamics; Finite element method; Liquid bridge; Filament stretching device; Viscoelasticity; Giesekus model; Moving boundary problem; POLYFLOW

\section{Introduction}

In a recent review [1] for the National Research Council on the future research trends in nonNewtonian fluid mechanics, Denn remarks that "There are two major outstanding problems in rheological measurement. One is the measurement of extensional stresses of mobile fluids such as polymer solutions, ... This measurement is essential for determining the predictive power of constitutive equations and the flow is closely related to many important processing situations." Pioneered by Matta and Tytus [2] and Sridhar et al. [3], the filament stretching device is one of the most promising experimental techniques that has been developed for providing accurate measurements of the transient extensional viscosity for polymer solutions. Numerous variants of such devices are currently being developed by research groups around the world [4-10].

In the filament stretching apparatus, a cylindrical liquid column is first generated between two concentric circular plates and then elongated by pulling one or both of the end-plate fixtures at an exponentially increasing rate. It is hoped that the resulting flow kinematics in the liquid column approximate an ideal uniaxial elongational flow; the extensional viscosity function is determined from the axial force at the end-plate which is measured as a function of time, and the total Hencky strain applied to the material is computed from the total stretch imposed on the sample [11].

As a result of the recent growing interest in filament stretching devices, theoretical and numerical efforts [12-18] have been devoted to studying the complex extensional behavior that viscoelastic liquids exhibit in such devices. Two classes of polymer solutions have been investigated so far: (i) dilute polymer solutions which exhibit pronounced strain-hardening (e.g. polystyrene (PS) or polyisobutylene (PIB)-based Boger fluids), and (ii) concentrated polymer solutions which only show weakly strain-hardening behavior, such as the test fluid that will be studied in the present work. It is found from both experimental and numerical studies that, under the same stretching conditions, the dynamical response of these two classes of viscoelastic fluids is dramatically different.

For a Boger fluid exhibiting a constant shear viscosity and a pronounced strain-hardening in uniaxial extension, the overall dynamical response of an elongating liquid in a filament stretching device is now fairly well understood. A summary of the key observations for this type of fluid has been given elsewhere [19]. Due to the increase in the transient extensional viscosity, the filament radius becomes progressively more axially uniform at large strains and hence leads to an increasingly homogeneous extensional deformation. As a result of this strain-hardening, the failure mechanism for the slender fluid column is dramatically different from the capillary-driven breakup observed in Newtonian fluid and jets which exhibit no strain-hardening [20-22]. In a filament stretching device, the large axial tensile stresses developed at large strains inhibit further elongation in the mid-region of the liquid bridge. Instead, the fluid reservoirs near either rigid end-plate are rapidly drained of fluid. The increasing curvature of the free surface in this region and the resulting gradients in the normal stresses lead to the onset of a local elastic instability [7]. Consequently, the stretching capability of the device (as characterized by the maximum achievable Hencky strain) is limited by the onset of a viscoelastic failure mechanism originating near the end-plates. 
For semi-dilute or concentrated polymer solutions, which typically exhibit shear-thinning viscometric properties and significantly less pronounced strain-hardening in extension, the current understanding of their extensional rheological behavior is still very limited. For such materials, the need for direct comparison between experiments and numerical simulations is more pressing [23] since the kinematics in the device never become spatially or temporally homogeneous [14,17]. Earlier experimental studies of some shear-thinning concentrated polymer solutions (e.g. the 'A1' and 'S1' fluids) were performed by Sridhar and co-workers [4,24]. Their measurements show that for these fluids, a strain-hardening extensional stress growth function still persists. Kolte et al. [17] performed computations with the Papanastasiou-Scriven-Macosko (PSM) integral model using material parameters fitted to rheological data for these fluids and showed that the stress growth measured in a filament stretching device agrees closely with the ideal extensional viscosity function expected in a homogeneous uniaxial elongation. For the values of the non-linear parameters selected in [17] for the PSM constitutive model, Trouton ratios of $\mathrm{O}(100)$ were obtained at moderate Hencky strains $(\epsilon \sim 4)$ and the radial profile of the elongating fluid column becomes increasingly uniform with time, in agreement with computations and experiments for strain-hardening Boger fluids.

However, in a recent study, Hassager et al. [25] show that as the parameter controlling the level of strain-hardening in the PSM model is varied and the ultimate steady-state extensional viscosity is gradually decreased, then the filament can undergo a ductile failure in which the radius of the filament rapidly decreases to zero at the axial mid-plane. This unstable necking phenomenon may be expected to severely compromise the ability of the filament stretching device to measure the extensional viscosity of weakly strain-hardening fluid such as polymer melts and concentrated polymer solutions. However, little experimental data for such materials has been available to date.

A detailed experimental and numerical study of the dynamical behavior of shear-thinning and weakly strain-hardening concentrated polymer solutions in filament stretching devices is reported by Yao, Spiegelberg and McKinley in [19]. The numerical simulations are coupled with experimental measurements using a $5.0 \mathrm{wt} \%$ concentrated solution of monodisperse polystyrene and show that, even for such fluids, it is possible to quantitatively measure the transient uniaxial extensional viscosity over a broad range of strain rates. Although the deformation in the elongating fluid filament is neither spatially nor temporally homogeneous when a single uniaxial stretching profile is applied to the end-plates of the device, accurate measurements of the tensile force and the rate of deformation of fluid elements near the mid-plane of the filament are sufficient to extract the transient extensional viscosity function. One important characteristic predicted by the simulations with the Giesekus model is that after an initial period of linear viscoelastic stress growth, the rate of necking in the fluid filament increases much more rapidly than is observed in either a corresponding Newtonian fluid or in an ideal elastic fluid (modeled by the Oldroyd-B constitutive equation). Consequently, this leads to the onset of unstable necking and the simulations suggest that the filament will eventually fail, or rupture in a finite time. In this case, the stretching capability of the device is restricted by the unstable necking developed in the middle of the filament. A generalized Considère criterion [19,25-27] can be used to help understand this unstable necking in the filament profile. This criterion can be obtained from energetic considerations of the static stability of an elastic column, and homogeneous elongation of a viscoelastic filament is unstable when the tensile force in the column passes through a maximum. However, the strain to failure and the rate of evolution of the neck is found to be a sensitive function of the dynamics of the chosen constitutive model $[19,25,28]$ 
The present study is a continuation of the work in [19] for the same experimental test fluid which exhibits strong shear-thinning in viscometric flows and very weak strain-hardening in extension. Smallamplitude oscillatory shear flow measurements for this fluid indicate that a broad spectrum of relaxation times is required to accurately model the response of the material [23], and consequently the dynamical evolution of a spatially non-homogeneous transient flow, such as the rapid necking in the filament radius, may be expected to be very sensitive to how accurately the constitutive response of the material is modeled. The numerical results presented in [19] are based primarily on two-dimensional, time-dependent simulations with the one-mode Giesekus model. In the present paper we extend these simulations to multi-mode formulations of the Giesekus constitutive equation and perform quantitative comparison of the numerical simulations and the experimental measurements.

Understanding the systematic modifications in the kinematics of a complex flow as the constitutive relationship for the fluid is varied, and detailed comparison between experiment and computation have been a central feature of numerous publications by Crochet and co-workers [29-31] and by other researchers [32-34]. Bodart and Crochet [35] have recently considered transient simulations of the sedimentation of a sphere accelerating from rest which, like a filament stretching device, is another complex flow containing regions of shear and extension. The importance of accurately determining the non-linear parameters in a constitutive model has been underscored by Satrape and Crochet [36], and, as in the present work, they document the dramatic difference in the kinematics that arises from simply changing the magnitude of the strain-hardening predicted in a region of strong extensional flow. In recent years, numerous groups have focused on achieving a quantitative comparison between experimental observations in complex viscoelastic flows and the associated linear stability analyses or transient numerical simulations $[23,37,44,45]$. These numerical studies emphasize the conclusion that it is necessary to accurately resolve both the linear viscoelastic spectrum and the non-linear response of the test fluid in large amplitude deformations through the use of multi-mode differential constitutive equations such as the Phan-Thien-Tanner and Giesekus models. The present work is a further contribution to this literature and we perform multi-mode time-dependent simulations using the numerical code POLYFLOW that has been developed by Crochet and co-workers over the past two decades.

We consider two types of deformation history commonly employed in filament stretching experiments. The first is a single-stage pure uniaxial extension of the type considered by numerous investigators; whilst the second is a two-stage deformation, consisting of pure uniaxial extension followed by a rapid cessation of elongation and the accompanying relaxation in the tensile stresses. The uniaxial elongation coupled with the no-slip boundary conditions at the two rigid end-plates results in a 'necked' configuration for any test fluid which will subsequently evolve after the external deformation is removed. However, one-dimensional (1-D) numerical simulations coupled with either finite element computations [22] or asymptotic analysis [20,21] have shown that the dynamical evolution of such an imperfection is a sensitive function of the chosen constitutive model and the resulting tensile force in the column. In particular, for the Oldroyd-B model, no necking failure is predicted, whereas for a fluid described by a Newtonian or Giesekus model the filament is predicted in each case to fail in finite time. In experiments with strongly strain-hardening Boger fluids that are described by the Oldroyd-B or FENE-P model, this viscoelastic stabilization of the fluid column has been exploited to permit investigation of the rapid and non-linear stress relaxation following cessation of uniaxial elongation [7,10,16,38]. It also forms the basis for a commercial device for extracting viscoelastic constitutive parameters from observations of filament breakup [39,40]. The primary goals of the present work are: 
to compare multi-mode simulations with experimental measurements and to contrast their predictive performance with analogous single-mode solutions; to study, through numerical simulation, the viscoelastic behavior of the shear-thinning weakly strain-hardening fluid during stress relaxation and to contrast the numerical calculations of the viscoelastic- and capillary-driven break-up processes with a $1-\mathrm{D}$ asymptotic analysis.

\section{Experimental background}

We consider the experimental measurements performed by Spiegelberg [19]. The test fluid used in the experiment is a $5.0 \mathrm{wt} \%$ solution of high molecular weight, narrow distribution polystyrene obtained from Pressure Chemical, dissolved in a mixture of tricresyl phosphate (TCP) and dioctyl phthalate (DOP). The preparation and viscometric characterization of the fluid is described by Li and Burghardt [23]. The steady-state shear viscosity and the first normal stress difference of the test fluid measured at a reference temperature of $22^{\circ} \mathrm{C}$ together with the predictions of the Giesekus model can be found elsewhere (e.g. Fig. 1 in [19]). Comparisons presented in [19,23] suggest that the experimental data can be well represented by a three-mode Giesekus model. The parametric values of the three-mode Giesekus model have been given elsewhere [19,23], but for completeness are listed again in Table 1. The three-mode fit was obtained by Li and Burghardt by first fitting the smallamplitude oscillatory shear data $\left(G^{\prime}(\omega), G^{\prime \prime}(\omega)\right)$ to obtain the linear viscoelastic parameters, $\eta_{i}$ and $\lambda_{i}$, then by adjusting the values of $\alpha_{i}$ for each mode to describe the first normal stress difference and the

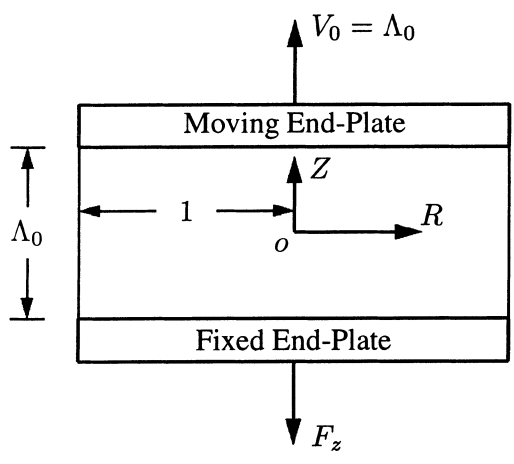

(a) $t=\varepsilon_{p}=0$

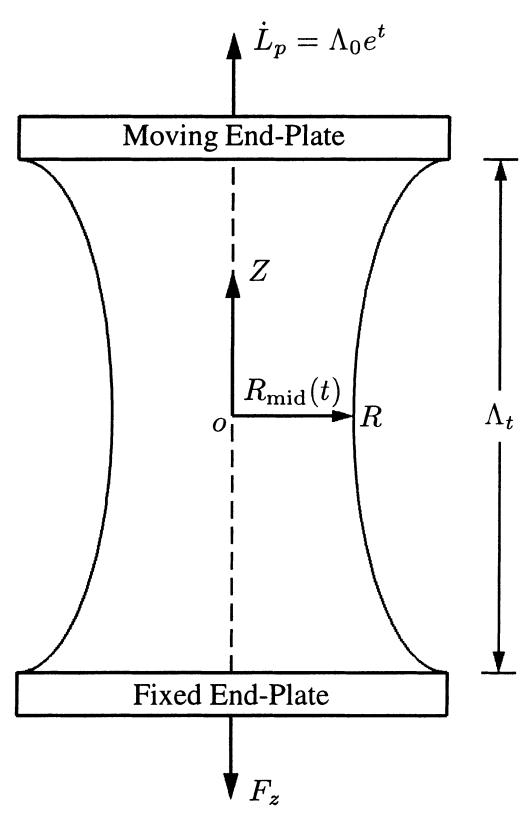

(b) $t=\varepsilon_{p}>0$

Fig. 1. Definition of the non-dimensional geometry and coordinate system for the liquid bridge in filament stretching devices. (a) Initial configuration of the cylindrical filament. (b) Subsequent extensional deformation. 
Table 1

Material properties of a weakly strain-hardening, $5 \mathrm{wt} \%$ solution of polystyrene and parameters of a three relaxation-mode Giesekus model fit given by Li and Burghardt [23]

\begin{tabular}{|c|c|c|c|c|}
\hline Parameter & Symbol [unit] & Mode 1 & Mode 2 & Mode 3 \\
\hline Relaxation time & $\lambda_{i}[\mathrm{~s}]$ & 0.421 & 0.0563 & 0.00306 \\
\hline Polymer viscosity & $\eta_{i}[\mathrm{~Pa} \mathrm{~s}]$ & 25.8 & 7.71 & 1.37 \\
\hline Mobility factor & $\alpha_{i}[-]$ & 0.3162 & 0.2422 & 0.0993 \\
\hline Density & $\rho\left[\mathrm{kg} / \mathrm{m}^{3}\right]$ & & 1030 & \\
\hline Solvent viscosity & $\eta_{s}[\mathrm{~Pa} \mathrm{~s}]$ & & 0.069 & \\
\hline Surface tension coefficient & $\sigma[\mathrm{N} / \mathrm{m}]$ & & 0.030 & \\
\hline
\end{tabular}

non-linear fluid response during start-up of steady shear flow. In our single-mode finite element simulations, we retain the values of $\eta_{1}$ and $\lambda_{1}$, and let $\lambda_{2} \rightarrow 0$ and $\lambda_{3} \rightarrow 0$ such that the total Newtonian solvent viscosity becomes $\eta_{\mathrm{s}}=0.069+\eta_{2}+\eta_{3}=9.15 \mathrm{~Pa}$ s. The solvent viscosity ratio for the single-mode model thus becomes $\beta=\eta_{\mathrm{s}} / \eta_{0}=0.262$. This value limits the extent of the shear-thinning in the viscosity and leads to deviation of the model predictions from measured data at high shear rates. In our two-mode calculations, we retain the parametric values of the first two modes and let $\lambda_{3} \rightarrow 0$. In this case, the Newtonian solvent viscosity becomes $\eta_{\mathrm{s}}=0.069+\eta_{3}=1.439 \mathrm{~Pa}$. The retardation parameter for the two-mode model then becomes much smaller, $\beta=\eta_{\mathrm{s}} / \eta_{0}=0.0412$.

The experiments were conducted using the filament stretching rheometer developed by Spiegelberg, Ables and McKinley [6]. A schematic diagram of the apparatus and discussion of the experimental subsystems can be found in [6]. For the experiments simulated in this work, the fluid sample is extruded through a positive-displacement syringe system onto rigid aluminium end-plates. The diameter of the circular end-plates used in this study was $d_{0}=2 R_{0}=0.7 \mathrm{~cm}$. The initial separation between the two endplates, $L_{0}$, is adjusted to ensure the sample configuration is initially cylindrical. The geometric parameters used in the tests are summarized in Table 2. During each filament stretching test, the lower plate is held stationary and is attached to a force transducer. A computer-controlled linear positioning system translates the upper end-plate so that the position and velocity of the end-plate both increase exponentially with time.

No velocity compensation algorithm of the type discussed in [4,6] is used in the experiments. The measured tensile force $F_{z}(t)$, corrected for surface tension and gravitational body force contributions

Table 2

Geometric parameters and non-dimensional numbers

\begin{tabular}{lllll}
\hline Parameter & Symbol [unit] & Test 1 & Test 2 & Test 3 \\
\hline Plate radius & $R_{0}[\mathrm{~m}]$ & 0.0035 & 0.0035 & 0.0035 \\
Initial aspect ratio & $\Lambda_{0}[-]$ & 0.583 & 0.629 & 0.54 \\
Extension rate & $\dot{E}\left[\mathrm{~s}^{-1}\right]$ & 2.32 & 3.42 & 4.48 \\
Reynolds number & $\mathrm{Re}=\rho \dot{E} R_{0}^{2} / \eta_{0}$ & $8.4 \times 10^{-4}$ & $1.2 \times 10^{-3}$ & $1.6 \times 10^{-3}$ \\
Capillary number & $\mathrm{Ca}=\eta_{0} \dot{E} R_{0} / \sigma$ & 9.46 & 13.94 & 18.27 \\
Bond number & $\mathrm{Bo}=\rho g R_{0}^{2} / \sigma$ & $\sim 0$ & $\sim 0$ & $\sim 0$ \\
Deborah number & $\mathrm{De}=\lambda_{1} \dot{E}$ & 0.98 & 1.44 & 1.89 \\
\hline
\end{tabular}


[6], and the filament radius, $R_{\text {mid }}(t)$ are then used to compute the evolution of the tensile stress difference in the column with time. For more details of the experimental procedures, readers are referred to [19].

\section{Computational background}

\subsection{Problem description}

Consider the extensional flow of a viscoelastic liquid contained between two parallel, coaxial, massless, equal-diameter, circular disks. The liquid column and the circular end-plates are the basic components of a filament stretching apparatus. This geometric configuration is also referred to as a liquid bridge [13] and is depicted schematically in Fig. 1. In this paper, we consider two different types of deformation history. The first is a one-stage process which involves uniaxial elongation only. The second is a two-stage process: uniaxial stretching followed by an instantaneous or very rapid deceleration and slower relaxation in the tensile stresses.

The initial configuration of the liquid bridge is assumed to be a cylinder when $t \leq 0^{-}$, as shown in Fig. 1(a). Let $R_{0}$ denote the radius of the two equal end-plates and $L_{0}$ the initial separation between the two end-plates. The initial aspect ratio of the liquid bridge is then defined as $\Lambda_{0} \equiv L_{0} / R_{0}$. At the instant $t=0^{+}$, the top plate is set into vertical motion with a prescribed velocity. A typical subsequent extensional deformation in the fluid filament is illustrated schematically in Fig. 1(b). The top plate will be referred to as the moving end-plate and its axial velocity is $\dot{L}_{\mathrm{p}}=\mathrm{d} L_{\mathrm{p}} / \mathrm{d} t$. The fluid column is assumed to remain axi-symmetric and to wet the end-plates so that the contact line is pinned to the radial edges of the disks at all times. The aspect ratio of the filament, $\Lambda_{t} \equiv L_{\mathrm{p}}(t) / R_{0}$, increases with time while the volume of the liquid bridge remains constant. In this study, we are particularly interested in the exponential separation between the two end-plates which is prescribed in dimensionless form by

$$
\Lambda_{t}=\Lambda_{0} \mathrm{e}^{t} \quad \text { and } \quad \dot{\Lambda}_{t}=\Lambda_{0} \mathrm{e}^{t}
$$

where $t$ is a non-dimensional time that will be defined in Section 3.2. With this scaling, the nondimensional initial velocity of the moving end-plate is $V_{0}=\Lambda_{0}$.

For the second type of deformation history, the uniaxial deformation prescribed above is performed up to a certain strain. The moving plate is then brought to a stop and the viscoelastic stresses in the filament begin to relax. In the computations, it is necessary to prescribe mathematically how the endplate decelerates to zero velocity. The following two assumptions are considered in the present work. The first assumes an instantaneous stop which can be considered as an idealized situation; the second assumes that the moving end-plate decelerates to zero velocity in a short time that corresponds to the finite ramping time in the real stretching apparatus. A typical ramping time for the current experimental design is about $50 \mathrm{~ms}$.

For the particular experiments considered in this work, the small size of the initial sample and the large viscous and elastic contributions to the total force ensure that gravitational body forces and inertia are negligible at all strains. In the present numerical simulations, the gravitational body force term in the momentum equation is neglected; however, for numerical reasons, the inertia term is retained in the calculations. 


\subsection{Governing equations}

To non-dimensionalize the governing equation, we select the radius of the end-plates, $R_{0}$, as a characteristic length, and use the imposed axial elongation rate $\dot{E}$ to construct both a time scale $(\dot{E})^{-1}$ and a viscous scale for the stress $\eta_{0} \dot{E}$. This leads to the following dimensionless variables:

$$
\begin{array}{ll}
r^{*}=r / R_{0} & z^{*}=z / R_{0} \\
\boldsymbol{u}^{*}=\boldsymbol{u} /\left(\dot{E} R_{0}\right) & t^{*}=t \dot{E} \\
p^{*}=p /\left(\eta_{0} \dot{E}\right) & \tau^{*}=\tau /\left(\eta_{0} \dot{E}\right)
\end{array}
$$

These variables along with the following dimensionless parameters

$$
\begin{array}{ll}
\text { Reynolds number } & \mathrm{Re}=\rho \dot{E} R_{0}^{2} / \eta_{0} \\
\text { Capillary number } & \mathrm{Ca}=\eta_{0} \dot{E} R_{0} / \sigma \\
\text { Deborah number } & \mathrm{De}_{i}=\lambda_{i} \dot{E}
\end{array}
$$

are used in obtaining the non-dimensional governing equations and boundary condition given below. Here $\eta_{0}$ is the zero-shear-rate viscosity, $\sigma$ is the surface tension coefficient and $\lambda_{i}$ is the relaxation time of the $i$ th mode. For convenience, we drop the asterisk notation in the remainder of the paper and do not explicitly identify variables as dimensionless. All the variables appearing below are assumed to be dimensionless, except two primary variables, $\dot{E}$ and $\eta_{0}$, which remain dimensional throughout the paper.

The fluid flow within the liquid bridge is assumed to be isothermal, incompressible, and axisymmetric, and is governed by the conservation equations for mass and linear momentum:

$$
\begin{aligned}
& \nabla \cdot \boldsymbol{u}=0 \\
& \operatorname{Re}\left(\frac{\partial \boldsymbol{u}}{\partial t}+\boldsymbol{u} \cdot \nabla \boldsymbol{u}\right)=\nabla \cdot \boldsymbol{\pi}
\end{aligned}
$$

Here $\boldsymbol{u}$ is the velocity vector and $\pi$ is the Cauchy stress tensor:

$$
\pi \equiv-p \boldsymbol{I}+\tau
$$

where $p$ is an isotropic pressure, $\boldsymbol{I}$ is the unit tensor and $\tau$ is the total extra stress tensor.

The appropriate boundary conditions for this problem include: the no-slip condition along the interface between the liquid and the end-plates, axi-symmetry along the $z$-axis, the motion of the moving end-plate prescribed by Eq. (1) and the following kinematic and dynamic conditions:

$$
\begin{aligned}
& \frac{\partial \mathscr{F}}{\partial t}+\boldsymbol{u} \cdot \nabla \mathscr{F}=0 \\
& \boldsymbol{\pi} \cdot \boldsymbol{n}=-\left(H / \mathrm{Ca}+p_{\mathrm{a}}\right) \boldsymbol{n}
\end{aligned}
$$

on the deformable free surface boundary. Eq. (7) is obtained by extending the formulation given in [22]. Here $\mathscr{F}(r, z, t)=r-R(z, t)=0$ is a function that defines the spatial position of the free surface $R(z, t)$, $\boldsymbol{n}$ is the unit norm of the surface, $p_{\mathrm{a}}$ is the ambient pressure, $H$ is the mean Gaussian curvature of the free surface and $\mathrm{Ca}$ is the Capillary number. Finally, at time $t=0^{-}$the following initial conditions for 
the velocity, pressure and extra stress fields are imposed,

$$
\boldsymbol{u}(r, z)=0, p(r, z)-p_{\mathrm{a}}=0 \quad \text { and } \quad \boldsymbol{\tau}(r, z)=\mathbf{0} \text { at } t \leq 0^{-}
$$

\subsection{Constitutive model}

To model the viscoelastic behavior of the test liquid considered in this work, we select the multimode Giesekus model [41], a non-linear differential constitutive equation based on the concept of deformation-dependent mobility. It can be derived from the Hookean dumbbell model [11,42] by allowing the Brownian motion and/or the hydrodynamic drag acting on the beads to be anisotropic. Previous numerical simulations have shown that the multi-mode Giesekus model is a good candidate for modeling polymeric flows $[32,43,44]$. In multi-mode formulations of this model, the solvent contribution $\tau_{\mathrm{s}}$ and the polymeric contribution $\tau_{\mathrm{p}}$ to the extra stress are defined as

$$
\begin{aligned}
& \boldsymbol{\tau}=\tau_{\mathrm{s}}+\tau_{\mathrm{p}} \\
& \tau_{\mathrm{s}}=2 \beta_{\mathrm{s}} \boldsymbol{D} \\
& \tau_{\mathrm{p}}=\sum_{i=1}^{n} \tau_{i} \\
& \tau_{i}+\mathrm{De}_{i} \tau_{i,(1)}+\frac{\alpha_{i} \mathrm{De}_{i}}{\beta_{i}} \tau_{i} \cdot \tau_{i}=2 \beta_{i} \boldsymbol{D} \\
& \tau_{i,(1)} \equiv \frac{\partial \tau_{i}}{\partial t}+\boldsymbol{u} \cdot \nabla \tau_{i}-(\nabla \boldsymbol{u})^{\mathrm{T}} \cdot \tau_{i}-\tau_{i} \cdot(\nabla \boldsymbol{u})
\end{aligned}
$$

where $\tau_{i,(1)}$ is the upper-convected time derivative of the extra stress tensor and the rate-of-strain tensor is defined as

$$
\boldsymbol{D} \equiv \frac{1}{2}\left[\nabla \boldsymbol{u}+(\nabla \boldsymbol{u})^{\mathrm{T}}\right]
$$

There are three independent physical parameters for each relaxation mode in this model; the polymer contribution of the $i$ th mode to the viscosity, $\eta_{i}$; the fluid relaxation time $\lambda_{i}$; and the dimensionless mobility factor $\alpha_{i}$ associated with anisotropic effects. Finally, we have an additional parameter $\eta_{\mathrm{s}}$ which originates from the contribution of the solvent to the total extra-stress tensor $\tau$. The zero-shear viscosity $\eta_{0}$ is obtained as the sum of all partial viscosity factors, i.e. $\eta_{0}=\eta_{\mathrm{s}}+\sum_{i}^{n} \eta_{i}$.

In the dimensionless Eqs. (11) and (13), we have $\beta_{i}=\eta_{i} / \eta_{0}, \beta_{\mathrm{s}}=\eta_{\mathrm{s}} / \eta_{0}$ and $\beta_{\mathrm{s}}+\sum_{i}^{n} \beta_{i}=1$. Eqs. (4)-(15) plus the boundary conditions form a set of governing equations for simulating the moving boundary problem of extensional deformation of viscoelastic liquid bridges.

For physically meaningful results with the Giesekus model, we require $0 \leq \alpha_{i} \leq 0.5$. In the limit $\alpha_{1} \rightarrow 0$ the single-mode Giesekus model reduces to the quasi-linear Oldroyd-B model and, in addition to predicting a constant shear viscosity $\eta_{0}=\eta_{\mathrm{s}}+\eta_{\mathrm{p}}$, the transient extensional viscosity function $\bar{\eta}^{+}$grows without bound for Deborah numbers De $=\lambda_{1} \dot{E} \geq 1 / 2$. As $\alpha_{i}$ is increased, the extent of the shearthinning in both the viscosity and the first normal stress difference increases while the value of the 
asymptotic or steady-state extensional viscosity decreases. For the values of $\alpha_{i}$ used in this work, the fluid is heavily shear-thinning and very weakly strain-hardening.

\subsection{Calculation of extensional viscosity}

The theoretical operating condition to be achieved in filament stretching devices is the ideal uniaxial elongational flow. In such a deformation, the local axial strain rate $\partial u_{z} / \partial z$ is homogeneous everywhere in the liquid and identical to the imposed global extension rate, $\dot{E}$. However, the kinematics in filament stretching devices are spatially non-homogeneous, as has been shown both experimentally [3,6] and numerically [14-17]. As a result, the local effective strain rate will vary throughout the elongating liquid column and the Hencky strain accumulated by material elements in the filament will be nonhomogeneous. For example, the Hencky strain based on the separation of the two end-plates, i.e.

$$
\epsilon_{\mathrm{p}}=t^{*} \equiv \dot{E} t=\ln \left(\Lambda_{t}\right)
$$

in general represents only the average of the local strain and strain rates experienced by all the fluid particles within the whole domain, as indicated by the first-order lubrication solution $[6,14]$. Note that with the chosen scaling, the axial or end-plate Hencky strain $\epsilon_{\mathrm{p}}$ is equivalent to the non-dimensional time.

Since the local extensional strain rate at the mid-plane is extremely important for the experimental data analysis, the following dimensionless effective extension rate is defined based on the free surface deformation at the mid-plane, $R_{\text {mid }}$,

$$
\dot{\epsilon}_{\mathrm{eff}} \equiv-2 \mathrm{~d}\left(\ln R_{\mathrm{mid}}\right) / \mathrm{d} t=-2 U_{\mathrm{r}, \mathrm{mid}} / R_{\mathrm{mid}}
$$

where $U_{\mathrm{r} \text {,mid }}$ is the radial velocity component of the free surface at the mid-plane. An effective Hencky strain based on $\dot{\epsilon}_{\text {eff }}$ can be calculated directly from Eq. (17) by the following integral,

$$
\epsilon_{\mathrm{eff}}=\int_{0}^{t} \dot{\epsilon}_{\mathrm{eff}}\left(t^{\prime}\right) \mathrm{d} t^{\prime}=-2 \ln \left[R_{\mathrm{mid}}(t)\right]
$$

If the deformation near the axial mid-plane is essentially uniform in the radial direction, then this effective Hencky strain represents the actual strain experienced by cylindrical fluid elements in the vicinity of the mid-plane.

For the ideal homogeneous uniaxial elongational flow, the transient Trouton ratio and its equivalent non-dimensional extensional viscosity function are defined as

$$
\operatorname{Tr}=\bar{\eta}^{+}(\dot{E}, t) \equiv \tau_{z z}-\tau_{r r}
$$

where $\tau_{z z}, \tau_{r r}$ are the normal components of the non-dimensional extra stress tensor defined in Eq. (10). In the ideal uniaxial elongational flow of a Newtonian fluid, the Trouton ratio is simply a constant with value $\operatorname{Tr}=3$.

In filament stretching devices, the time-dependent quantities to be measured experimentally are the normal force on the end-plate, $F_{z}(t)$, and the mid-plane radius of the fluid filament, $R_{\text {mid }}(t)$. From these experimental measurements, it is necessary to compute the extra stress difference and the extension rate 
in the fluid. Since these quantities vary spatially throughout the material, a number of different formulae for the 'apparent extensional viscosity' measured by the device have thus been proposed in the literature. Following the notation used in Refs. [17,18] we define two different quantities appropriate for an experiment in which a single exponential velocity profile of the form given in Eq. (1) is imposed. In a Type $I$ experiment the imposed axial extension rate and the average, or idealized, filament radius are used to compute the extensional viscosity function from the experimentally measured force,

$$
\bar{\eta}_{(\mathrm{I})}^{+}\left(\epsilon_{\mathrm{p}}\right)=\frac{F_{z}}{\pi R_{\text {ideal }}^{2}}-\frac{1}{\mathrm{Ca} R_{\text {ideal }}}+\mathrm{O}\left(F_{\mathrm{i}}, F_{\mathrm{g}}\right)
$$

where $R_{\text {ideal }}=\exp \left(-0.5 \epsilon_{\mathrm{p}}\right)$ is the non-dimensional ideal radial deformation in uniaxial elongational flow. Note that the imposed axial extension rate does not appear explicitly in Eq. (20a), this is because the extension rate is scaled by itself and hence we have $\dot{E} \equiv 1$ in its dimensionless form. In the above expression, the second term on the right hand side is the correction term arising from capillary effects with non-zero surface tension, $\sigma$. The final term $\mathrm{O}\left(F_{\mathrm{i}}, F_{\mathrm{g}}\right)$ accounts for the corrections due to the inertia force $F_{\mathrm{i}}$ and gravitational force $F_{\mathrm{g}}$, respectively. In the results presented in this paper, this last term is assumed to be negligibly small. Detailed studies of the inertia and gravity corrections will be pursued in later publications.

The use of $R_{\text {ideal }}$ in Eq. (20a) implies that in the original type I analysis the filament is assumed to deform homogeneously as a cylinder throughout the test. This assumption is clearly a poor one, especially at early times (i.e. small strains), and modification is made in practice by making use of additional experimental knowledge of the evolution of the filament radius $R_{\text {mid }}(t)$ at the axial mid-plane $z=\Lambda_{t} / 2$. There are several ways in which this additional information can be utilized. Firstly, the actual mid-point filament radius can be substituted in Eq. (20a) to provide a better estimate of the tensile stress in the filament. We refer to this as a modified type I or type IB analysis:

$$
\bar{\eta}_{(\mathrm{IB})}^{+}\left(\epsilon_{\mathrm{p}}\right)=\frac{F_{z}}{\pi R_{\mathrm{mid}}^{2}}-\frac{1}{\mathrm{Ca} R_{\text {mid }}}+\mathrm{O}\left(F_{\mathrm{i}}, F_{\mathrm{g}}\right)
$$

The experimental data presented in the present work are analyzed using this type IB formulation.

A further improvement for extensional viscosity calculations is to replace the constant axial extension rate, $\dot{E}$, in Eq. (20b) by the time-dependent local effective extension rate, $\dot{\epsilon}_{\text {eff }}$, defined in Eq. (17). This procedure leads to the Type II formula defined in Ref. [17]:

$$
\bar{\eta}_{(\mathrm{II})}^{+}\left(\epsilon_{\mathrm{eff}}\right)=\frac{F_{z}}{\dot{\epsilon}_{\mathrm{eff}} \pi R_{\mathrm{mid}}^{2}}-\frac{1}{\mathrm{Ca} \dot{\epsilon}_{\mathrm{eff}} R_{\mathrm{mid}}}+\mathrm{O}\left(F_{\mathrm{i}}, F_{\mathrm{g}}\right)
$$

For clarity, the type II analysis is used exclusively in the present work for post-processing numerical solutions and for comparing with the type IB analysis.

Note that the use of local variables in evaluating the kinematics in Eq. (21) means that the computed Trouton ratio pertains specifically to the material elements near the mid-plane, even though the stress difference is measured through the force transducer located at the rigid end-plate. Similar problem are faced in analysis of other extensional flow devices; however, the benefit of the filament stretching device is that, although the deformation in the filament is spatially non-homogeneous throughout the experiment, the tensile force transmitted axially along column is constant at any instant in time for all 
$0 \leq z \leq \Lambda_{t}$ (assuming inertial forces are negligibly small). By symmetry, shearing deformation are negligible at the axial mid-plane, except for very short times or small aspect ratios and Eq. (21) thus should accurately capture the response of a material element to a homogeneous uniaxial elongational flow. In the present work we investigate the applicability of the type II analysis by comparing the experimental measurements with numerical predictions and with the theoretically computed Trouton ratio expected in an ideal homogeneous uniaxial elongation.

\subsection{Numerical algorithms}

The set of governing equations presented in Sections 3.2 and 3.3 will be solved by means of the finite element technique [46]. For this, we use the commercial finite element package POLYFLOW primarily designed for the analysis of industrial flow situations dominated by non-linear viscous phenomena and viscoelastic effects. Details on the available applications and on the numerical technique used in POLYFLOW are document in $[47,48]$.

Several finite element algorithms are available in the literature. Their respective properties and performances have been reviewed and detailed (see for example in [49-52]). Over the last two decades, these algorithms have been steadily improved and applied with increasing efficiency to both confined and free surface flows.

In the present paper, we employ the finite element algorithm originally developed by Kawahara and Takeushi [53] and referred to as the MIX1 method [46,49]. In this method, all viscoelastic extra stresses, velocity and pressure unknown fields are approximated by means of finite expansions. The Galerkin formulation is then invoked for discretizing the flow governing equations. In the early 1980s, this technique permitted simulation results to be obtained for the extrudate swell and the fiber drawing of viscoelastic fluids [54-57].

This finite element method MIX1, is readily extended to multi-mode differential viscoelastic models, and all viscoelastic extra-stress components $\tau_{i}$ and the velocity $\boldsymbol{u}$ are interpolated by means of biquadratic shape functions. The pressure $p$ is represented by means of independent first-degree polynomials in each finite element instead of bilinear shape functions. This interpolation enforces the local solenoidal character of the velocity field by strictly imposing the mass conservation across the boundary of each element. This has significantly improved the performances of the MIX1 method for Poiseuille flow of a Johnson-Segalman fluid [58] and for the contraction flow of a Phan-Thien-Tanner fluid [59]. A further improvement has been developed by Crochet and co-workers [60,61], which consists of using bilinear sub-element interpolation for the viscoelastic extra-stresses together with a streamline upwinding technique. However, the required computer resources are relatively high for the simulation of a viscoelastic flow with a relaxation spectrum [62] and may be prohibitive for a timedependent calculation in this context.

Keunings et al. [63,64] have extended the MIX1 method to time-dependent viscoelastic flows involving moving boundaries. In particular, they have applied their technique to studies of jet break-up [22]. By invoking the Galerkin formulation on deforming finite elements [65], one obtains a set of firstorder ordinary differential equations which are then discretized in time by mean of standard techniques [66]. The technique has been successfully applied to several flow situations (see e.g. [22,63,64,67-69]). The transient problem is solved by means of a predictor-corrector integration scheme in which the corrector is the backward Euler method. The time-step is controlled using the algorithm suggested by Gresho et al. [70,71]. 
The governing equations and free surface conditions are solved in a coupled fashion. At each time step, the non-linear algebraic system resulting from the finite element discretization is solved by Newton-Raphon iteration. Termination of the non-linear iteration is controlled by a specific iteration convergence criterion of $10^{-5}$ for the relative error norms of the residuals of the governing equations and the free surface update. Spatial and temporal convergence of the simulation have been verified separately.

Another important aspect for moving boundary problems is the remeshing algorithm which controls mesh deformations by relocating internal nodes according to the displacement of boundary nodes, in order to avoid unacceptable element distortions. A remeshing rule based on the Thompson transformation is used in this work [72]. It consists of solving a partial differential equation of elliptic type for the coordinates, and it exhibits a high robustness even for very large mesh deformations.

The numerical model used in this study is axi-symmetric and assumes solution symmetry with respect to the axial mid-plane between both end-plates. Consequently, the computational domain is defined by $0 \leq r \leq R(z, t)$ and $0 \leq z \leq \Lambda_{t} / 2$ and we refer to this configuration as the half length model. The computational domain is discretized by means of finite elements as displayed in an intermediate configuration in Fig. 2. The mesh contains 720 quadrilateral elements and 793 vertices. For the three-mode simulations investigated in the present work, this typically leads to 48600 degrees of freedom including unknowns of velocity, pressure, polymer stress and free surface coordinate. The total number of time steps required to achieve a strain of $\epsilon_{\mathrm{p}} \sim 4$ is about 800. The computations are performed on a Silicon Graphics four-processor IRIX Power Challenge (MIPS R8000) machine. In the present context, the parallel capabilities of the computer are exploited at the level of basic linear algebra

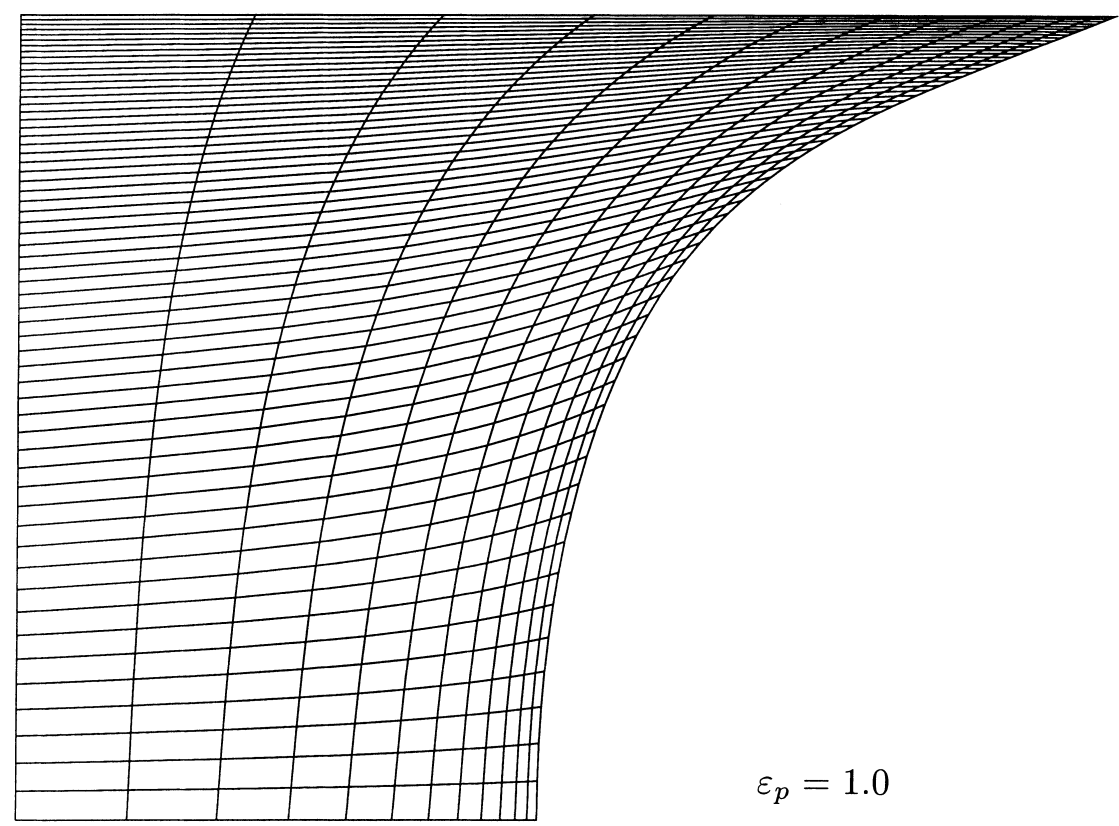

Fig. 2. Deformed finite element mesh at a strain of $\epsilon_{\mathrm{p}}=1.0$. The top boundary is the moving end-plate $z=\Lambda_{t} / 2$, the bottom boundary is the mid-plane $z=0$, the left vertical boundary is the symmetric axis $r=0$ and the right boundary curve is the free surface. 
for performing the several matrix-vector products in order to solve the system of unknowns. In those circumstances, a typical computation takes approximately $32 \mathrm{~h}$ CPU time.

\section{Extensional deformation}

In this section, we present numerical results for the weakly strain-hardening test fluid, a $5.0 \mathrm{wt} \%$ concentrated polystyrene solution described in Section 2. Numerical solutions are obtained using the multi-mode Giesekus model. The predicted transient Trouton ratios are compared with experimental measurements as well as theoretical results. To examine the differences in the numerically predicted viscoelastic behavior and the role of the relaxation spectrum, we also present a detailed comparison of multi-mode solutions with single-mode solutions. In addition, a Newtonian fluid with the same solvent viscosity as that of the test liquid will serve as a reference for comparison purposes.

The basic geometric parameters and the material properties used in the simulation are given in the Tables 1 and 2. All results are presented in non-dimensional form, in which the dimensionless time is equivalent to the Hencky strain $\epsilon_{\mathrm{p}}$ defined in Eq. (16).

\subsection{Multi-mode solutions vs. single-mode solutions}

We first examine the differences in the fluid kinematics and dynamics predicted by the multi-mode and single-mode formulations. Among the three experimental tests summarized in Table 2, we choose the third test with $\mathrm{De}=1.89$ as an example. Three solutions are computed for this test using the onemode, two-mode and three-mode Giesekus models, respectively. The material parameters for the threemode fit and the geometric information are given in the Tables 1 and 2.

Since the single-mode model incorporates a much larger response from the solvent viscosity, it is expected that multi-mode solutions will differ significantly from the one-mode solution at short times. This can be easily seen from the comparison of the simulated axial tensile force $F_{z}$ at the mid-plane presented in Fig. 3. As we can see from Fig. 3(a), the one-mode solution has a much higher initial value of $F_{z}$. This is simply due to the fact that the initial dynamical response is dominated by the contribution from the Newtonian solvent. In the absence of a large viscous contribution, the axial force in the multimode simulations is governed by linear viscoelastic growth of the polymeric stresses. This difference leads to a significant difference in the predicted extensional viscosity. The results in Fig. 3(a) also show that the forces predicted by the two-mode and the three-mode solution are almost identical. This is because the third-mode has a much shorter relaxation time $\left(\lambda_{3} / \lambda_{2} \approx 1 / 18\right)$ and also a much smaller viscosity factor $\left(\eta_{3} / \eta_{2} \approx 1 / 5.6\right)$. Hence the individual contribution of the third-mode to the extensional viscosity develops faster but remains small as compared to that of the other modes. It is interesting to note that the multi-mode solutions lead to a local maximum in the $F_{z}$-curve. According to the generalized Considère criterion given in $[19,25]$, the local maximum signifies that unstable necking will occur during further stretching and the location of the local maximum provides an estimate of the critical strain. For completeness, we also show here a numerical convergence check for the multi-mode solution by considering two meshes, a $10 \times 40$ finite element grid with 10 elements in radial direction and a $12 \times 60$ grid. In Fig. 3(b) we compare the calculated axial tensile force at the mid-plane based on the two meshes. The difference between the two solutions is very small. However, for better accuracy, the solutions presented in this work are all based on the finer mesh. 

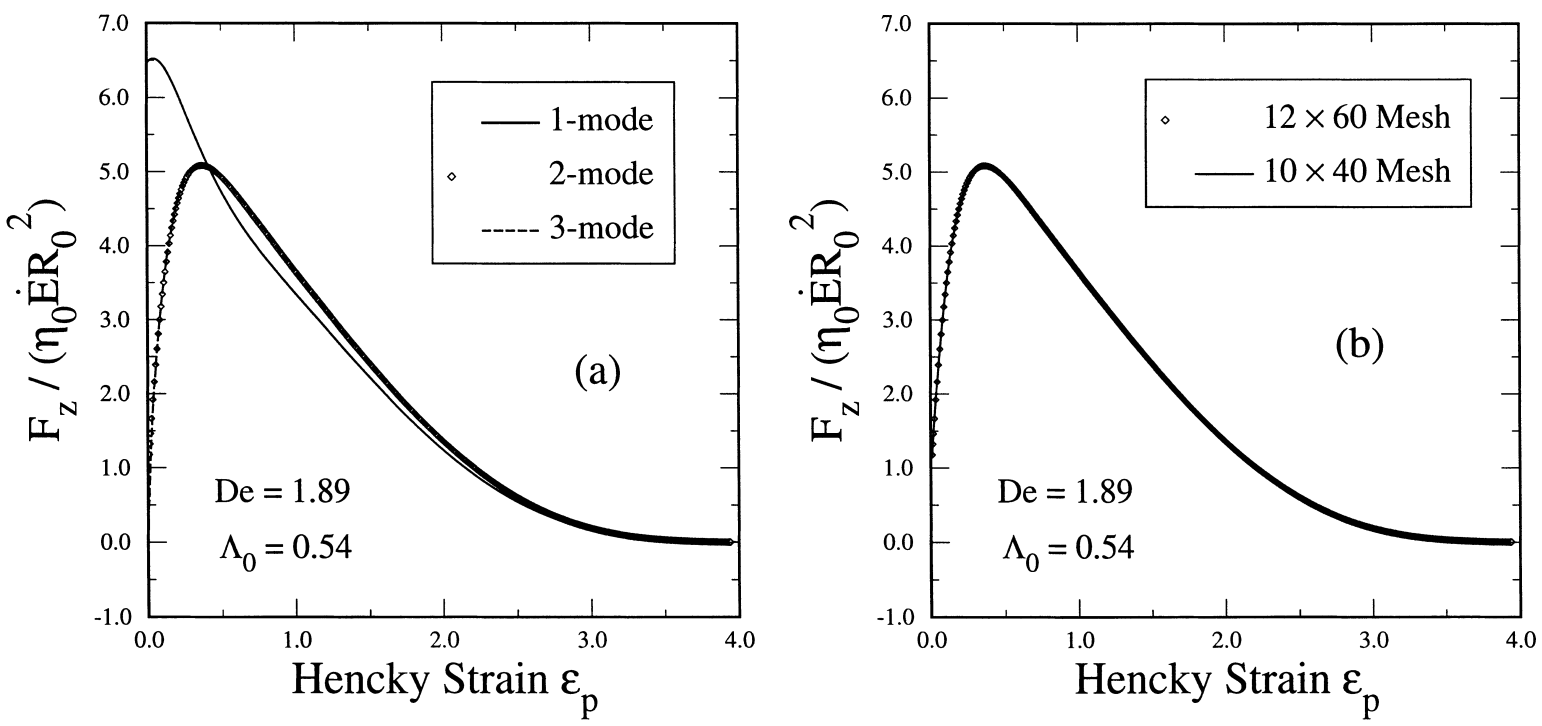

Fig. 3. Variation of the axial tensile force at the mid-plane between two end-plates as a function of Hencky strain $\epsilon_{\mathrm{p}}$. The relevant parameters used in this example are $\mathrm{De}=1.89,1 / \mathrm{Ca}=0.0524, \Lambda_{0}=0.54$. (a) Comparison of single-mode solution with multi-mode solutions; (b) effect of mesh refinement.

Next we examine the flow kinematics characterized by the effective extensional strain rate $\dot{\epsilon}_{\text {eff }}$ defined in Eq. (17). It has been shown elsewhere [14,19] that $\dot{\epsilon}_{\text {eff }}$ can be used to accurately characterize the actual extensional strain rate that fluid elements experience at the mid-plane. In Fig. 4 the simulated effective extension rate is plotted as a function of Hencky strain $\epsilon_{\mathrm{p}}$. There are two regions where the multi-mode solutions differ from the single-mode solution. The first region is the small strain range $0.05<\epsilon_{\mathrm{p}}<1.0$. An enlarged view for this region is shown in Fig. 4(b). When $\epsilon_{\mathrm{p}}>0.05$, the multimode solutions start to differ from the one-mode solution and exhibit a lower extension rate, indicating that the multi-mode solution exhibit a slightly stronger strain-hardening due to the polymer stress contributions from the higher relaxation modes. The maximum difference (only about $4 \%$ ) between the multi-mode and one-mode solutions in this region occurs at $\epsilon_{\mathrm{p}} \approx 0.25$. The difference decreases when $\epsilon_{\mathrm{p}}>0.25$ and becomes negligibly small when $\epsilon_{\mathrm{p}}>1.0$. The results in this region also suggest that the contribution to strain-hardening from the third relaxation mode is negligibly small as compared to the second-mode contribution. The second region in which the single-mode results differ is the large strain range $\epsilon_{\mathrm{p}}>2.5$. In this region, the multi-mode solutions predict a faster necking rate than the singlemode solution. This arises since the higher viscoelastic modes provide less instantaneous viscous resistance to the elastic recoil of the macro-molecules near the end-plates and hence calculations predict an even more accelerated unstable necking than the one-mode simulation. Consequently, analysis of the necking failure based on the one-mode calculation can be considered as a more conservative estimate of the time to break-up.

To investigate spatial differences in the fluid kinematics, in Fig. 5 we plot axial profiles of the individual components of the rate-of-deformation tensor defined in Eq. (15). Two typical strain levels are selected from consideration of the effective extensional strain rate shown in Fig. 4 . The first is $\epsilon_{\mathrm{p}}=0.25$ where there is a local maximal difference in $\dot{\epsilon}_{\mathrm{eff}}$ between the multi-mode and single-mode 

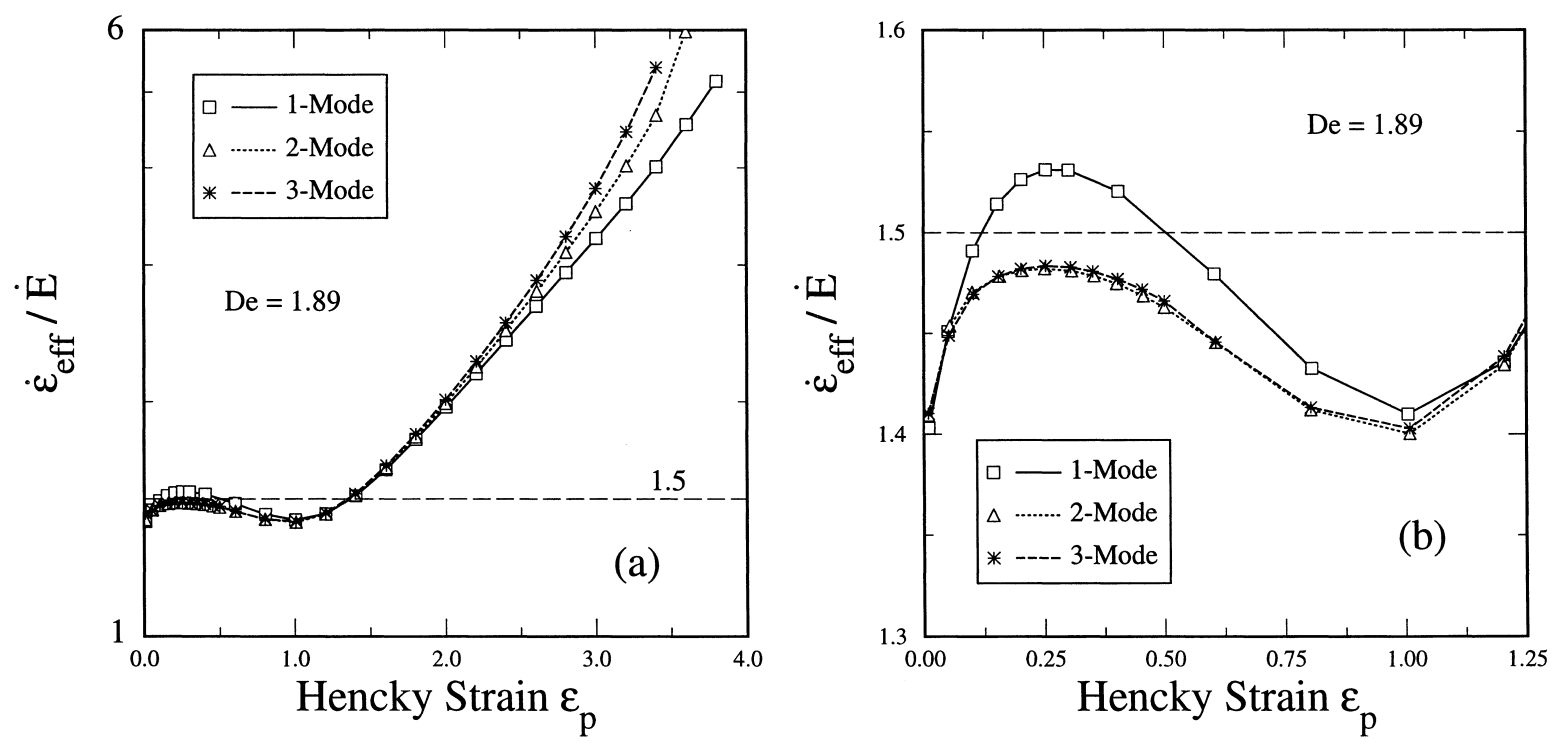

Fig. 4. The role of multi-mode calculations in predicting the flow kinematics characterized by the effective extension rate $\epsilon_{\text {eff }}$ defined in Eq. (17). (a) Plotted in semi-logarithm scale; (b) local enlargement in linear scale for the small strain range.

solutions. Fig. 5(a) shows the variation of the axial velocity gradient $D_{z z}$ along the centerline of the filament. The difference between the multi-mode and one-mode solutions is very small along the centerline. The results suggest that the simple lubrication squeeze-flow solution, originally derived for Newtonian fluids with small initial aspect ratios, also provides a good approximation of the kinematics for the multi-mode Giesekus model at small strains. In Fig. 5(b) we plot the spatial variation of the radial component of the rate-of-deformation tensor $D_{r r}$ on the free surface for $\epsilon_{\mathrm{p}}=0.25$. Once again the two-mode and three-mode solutions are almost identical. The multi-mode solutions differ from the single-mode solution primarily in two regions: the central part (around $z=0$ ) and a small region near the rigid end-plate. In the central region, the absolute value of $D_{r r}$ for the one-mode solution is larger than that for the multi-mode solution, indicating that the radial contraction rate at the central portion of the free surface predicted by the multi-mode model is slightly lower than that of the one-mode model. This slight decrease in the free surface deformation in multi-mode computations is consistent with the effective extension rate shown in Fig. 4. The second strain level selected for comparison is $\epsilon_{\mathrm{p}}=3.0$. In Fig. 5(c) we compare the profiles of $D_{z z}$ along the centerline at this large strain. The results in Fig. 5(c) demonstrate the major difference between the multi-mode and single-mode solutions, namely the multi-mode solutions predict a significantly stronger elastic recoil of the fluid near the end-plate (as indicated by larger negative values of $D_{z z}$ ). As a result, the multi-mode predicts a higher local extension rate near the mid-plane, which leads to a more rapid necking failure.

In Fig. 6 we examine the polymer stress growth for the three-mode solution. The axial stress components of $\tau_{i, z z},(i=1,2,3)$ are computed pointwise at the center of the filament, $r=z=0$. In Fig. 6(a) we scale the stress by $\eta_{0} \dot{E}$ and plot the stress as a function of $\epsilon_{\mathrm{p}}$. The theoretical curves are obtained using the ideal flow kinematics in the uniaxial elongational flow. As we can see, the calculated stress does not agree with theory: in particular, it does not approach a steady-state. This is because the actual kinematics within the elongating filament are non-homogeneous both spatially and temporally. 

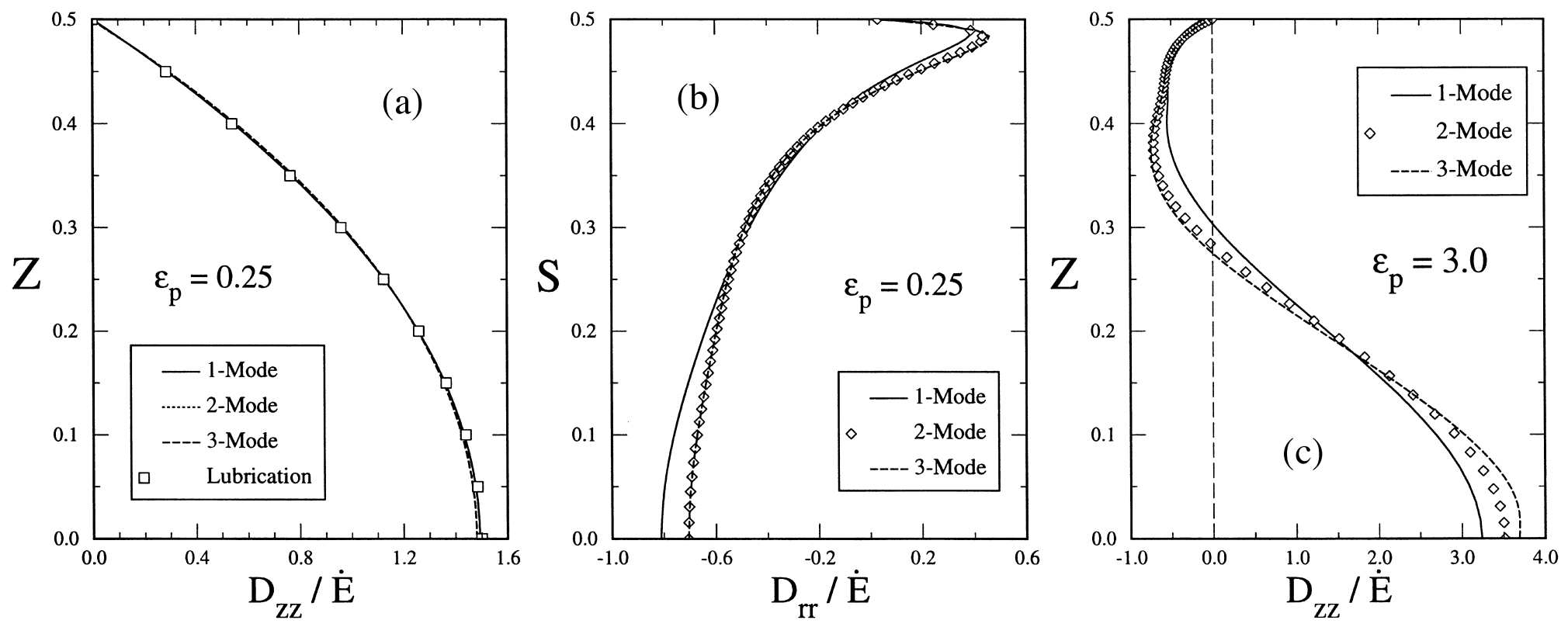

Fig. 5. A quantitative comparison of the flow kinematics at two selected strain levels. The extensional flow kinematics are characterized by the rate of deformation tensor $\boldsymbol{D}$ defined in Eq. (15). Here the axial-coordinate is scaled by the separation length $L_{\mathrm{p}}(t)$ between the two end-plates, while $S$ is scaled by the curvilinear length of the free surface. $z=S=0$ corresponds to the mid-plane and $z=S=0.5$ to the upper moving end-plate. (a) The axial velocity gradient $D_{z z}$ along the centerline $r=0$ at a strain of $\epsilon_{\mathrm{p}}=0.25$; (b) the radial velocity gradient $D_{r r}$ on the free surface at $\epsilon_{\mathrm{p}}=0.25$; (c) $D_{z z}$ along the centerline at $\epsilon_{\mathrm{p}}=3.0$. 

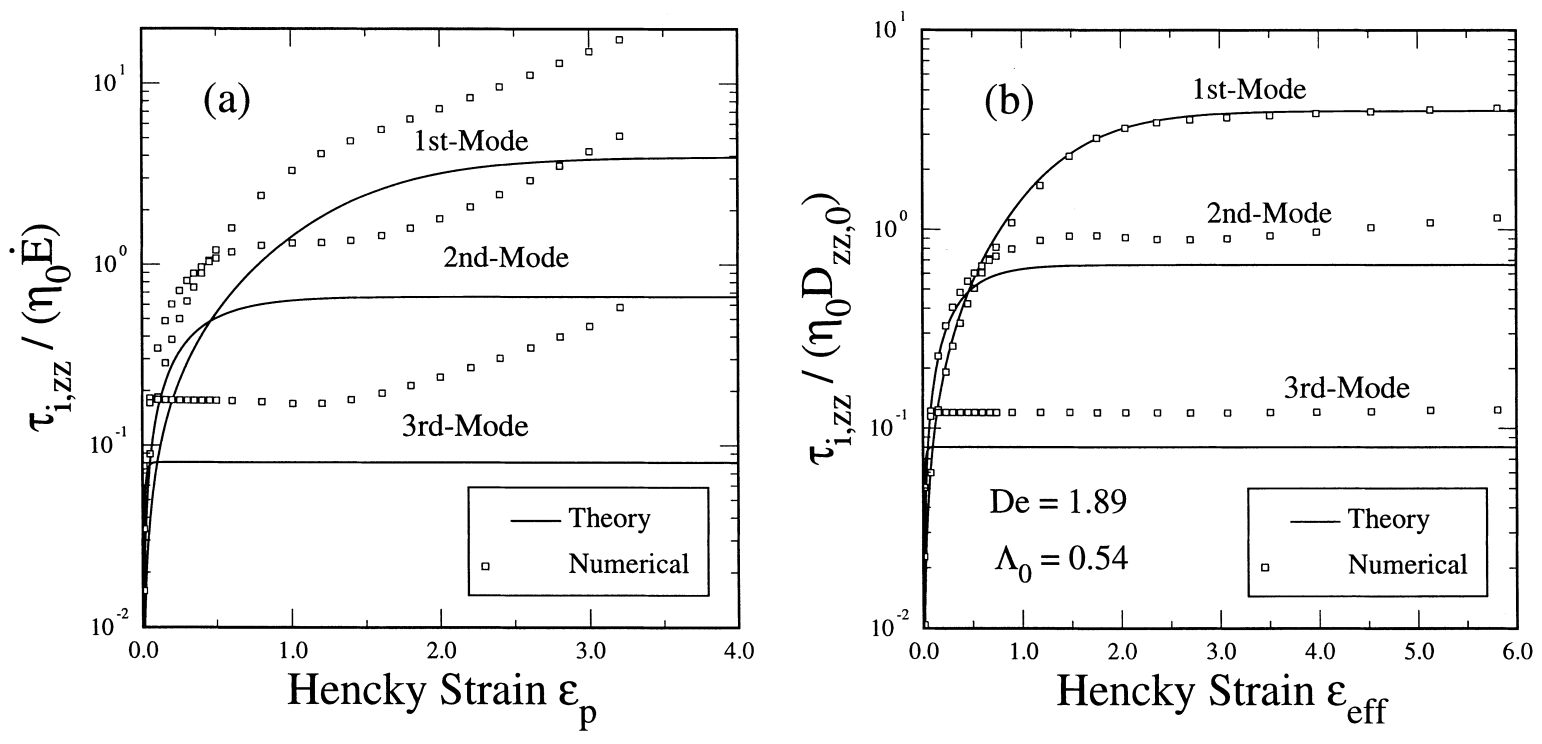

Fig. 6. Time history of the axial component of polymeric stress contributions for the three-mode Giesekus model solution. The stress is evaluated pointwise at the center of the liquid bridge, $r=0$ and $z=0$. (a) $\tau_{i, z z} /\left(\eta_{0} \dot{E}\right.$ ) as a function of $\epsilon_{\mathrm{p}}$; (b) $\tau_{i, z z} /\left(\eta_{0} D_{z z}\right)$ as a function of $\epsilon_{\mathrm{eff}}$.

For example, the actual local extensional strain rate at the mid-plane varies with time and becomes significantly higher than the imposed constant extension rate $\dot{E}$. As a result, the actual local strain at the mid-plane is much larger than the average strain $\epsilon_{\mathrm{p}}$. To improve the analysis, we re-scale the stress by $\eta_{0} D_{z z}(0,0, t)$ and plot it as a function of $\epsilon_{\text {eff. }}$. Here $D_{z z}(0,0, t)$ is the pointwise value of $D_{z z}$ at the centre of the filament, and the effective strain $\epsilon_{\text {eff }}$ is calculated using the integral $\int_{0}^{t} D_{z z}\left(0,0, t^{\prime}\right) \mathrm{d} t^{\prime}$. Therefore, the spatial and temporal non-homogeneity experienced by the fluid particle at the center point is now accounted for by the new scale and the new variable $\epsilon_{\text {eff. }}$. The rescaled stresses presented in Fig. 6(b) show a much better agreement with theory. The agreement for the first mode is excellent. The results presented in Fig. 6 provide an important insight for experimental measurement: in addition to the force measurement, an accurate determination of the local kinematics is essential for more accurate interpretation of experimental data, due to the highly non-homogeneous deformation generated in filament stretching devices.

\subsection{Extensional viscosity}

We now consider the three experimental tests described in Section 2 and Table 2 and compare the simulated transient extensional viscosity with the experimental measurements in [19] and with theoretical predictions for homogeneous uniaxial elongation. The experimental observables are the total normal force at the lower end-plate, $F_{z}$, and the free surface profile $R(z, t)$ from which the mid-plane radius, $R_{\text {mid }}(t)$, can be extracted. These experimental data are then used to calculated the transient extensional viscosity based on the type IB formulation described in Eq. (20b).

We first consider the third test run with an imposed extension rate $\dot{E}=4.68 \mathrm{~s}^{-1}$ and a Deborah number $\mathrm{De}_{1}=1.89$. Three numerical solution are obtained using the one-mode, two-mode and three- 

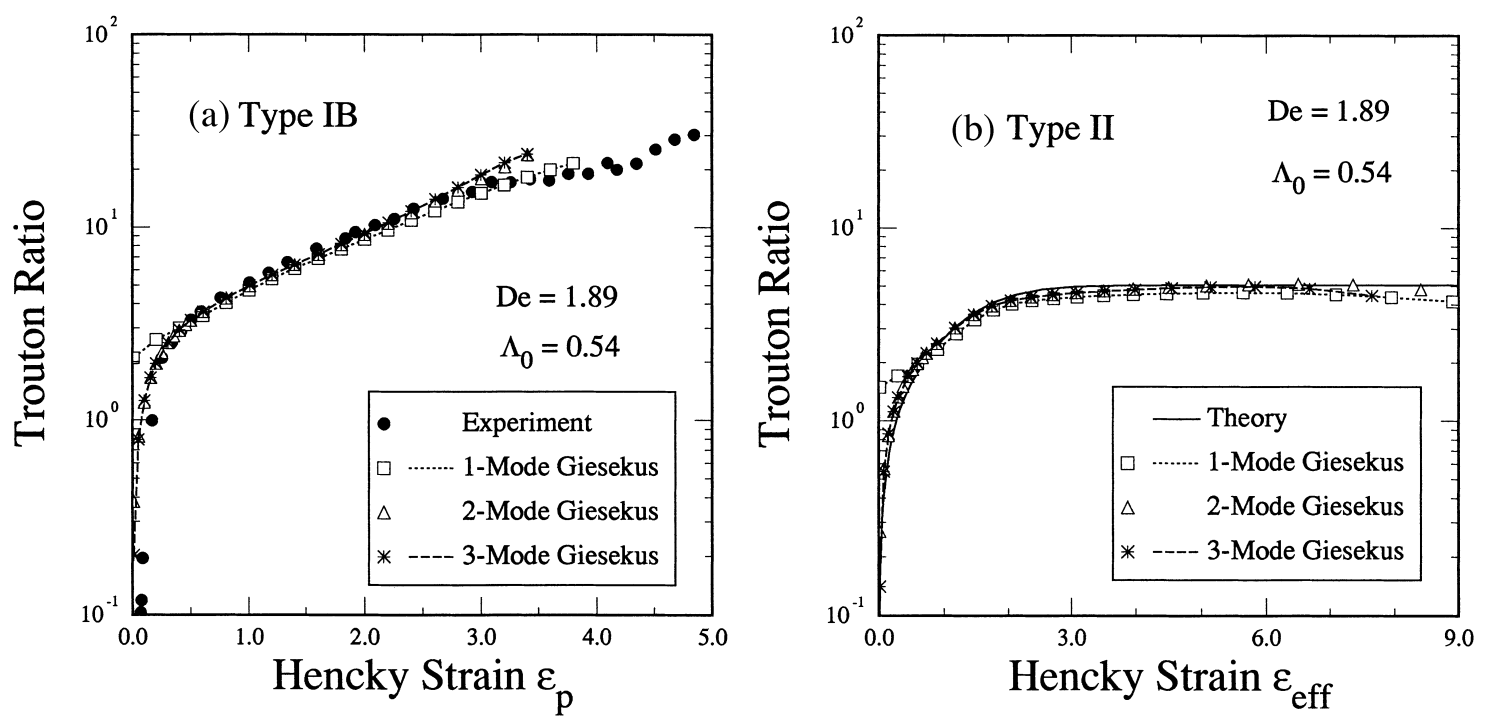

Fig. 7. Comparison of the simulated Trouton ratio and with the experimental data for test run 3 with $\mathrm{De}=1.89$ and $\Lambda_{0}=0.54$. The three numerical solutions are obtained using the one-mode, two-mode and three-mode Giesekus models, respectively. (a) Comparison based on the type IB analysis defined in Eq. (20b). (b) Based on the type II analysis defined in Eq. (21). The curve denoted 'theory' refers to the analytical solution for the three-mode Giesekus model under homogeneous uniaxial elongational flow conditions.

mode models, respectively, and the simulated transient Trouton ratios are presented in Fig. 7. In Fig. 7(a) the measured transient extensional viscosity $\bar{\eta}_{(\mathrm{IB})}^{+}$is plotted as a function of the axial Hencky strain $\epsilon_{\mathrm{p}}$ defined by Eq. (16). The three numerical solution shown in Fig. 7(a) are also post-processed using the same 'type IB' analysis. For the single-mode model, the agreement between measurement and simulation is excellent except for the initial small strain range. This discrepancy at short times arises mainly from the following two sources. Firstly, in the experiments, there is a finite ramping time (approximately $0.05 \mathrm{~s}$ for the present device) required for the motor to accelerate from rest state to its initial velocity, whereas in numerical calculations it is assumed that the moving plate attains its initial velocity $V_{0}=\Lambda_{0}$ instantaneously. Secondly, for the small initial aspect ratios used in the tests, the error in the measured force induced by additional shearing deformation near the rigid end-plates is artificially elevated during the initial stage, due to the larger initial solvent viscosity in the single-mode calculation. This second error source is greatly reduced when the multiple relaxation modes of the fluid are incorporated. In Fig. 7(b), the numerical solutions are also post-processed using the type II analysis defined in Eq. (21) and the results are compared with the theoretical prediction assuming ideal uniaxial elongational flow kinematics. Note that here the curve denoted 'theory' is based on the three-mode Giesekus model. As we can see the multi-mode calculation provides a much better prediction for the initial stress growth of the material within the small strain range $\epsilon_{\mathrm{p}} \leq 0.6$, and compares very favourably to both experimental measurement and theoretical prediction. The difference between the 2-mode and three-mode solutions is very small. The comparison presented in Fig. 7 suggests that the two-mode calculation can provide adequate accuracy at a relatively lower computational cost for most of the Hencky strains examined in the present work. For this reason, the other two experimental tests are simulated using the two-mode Giesekus model. 

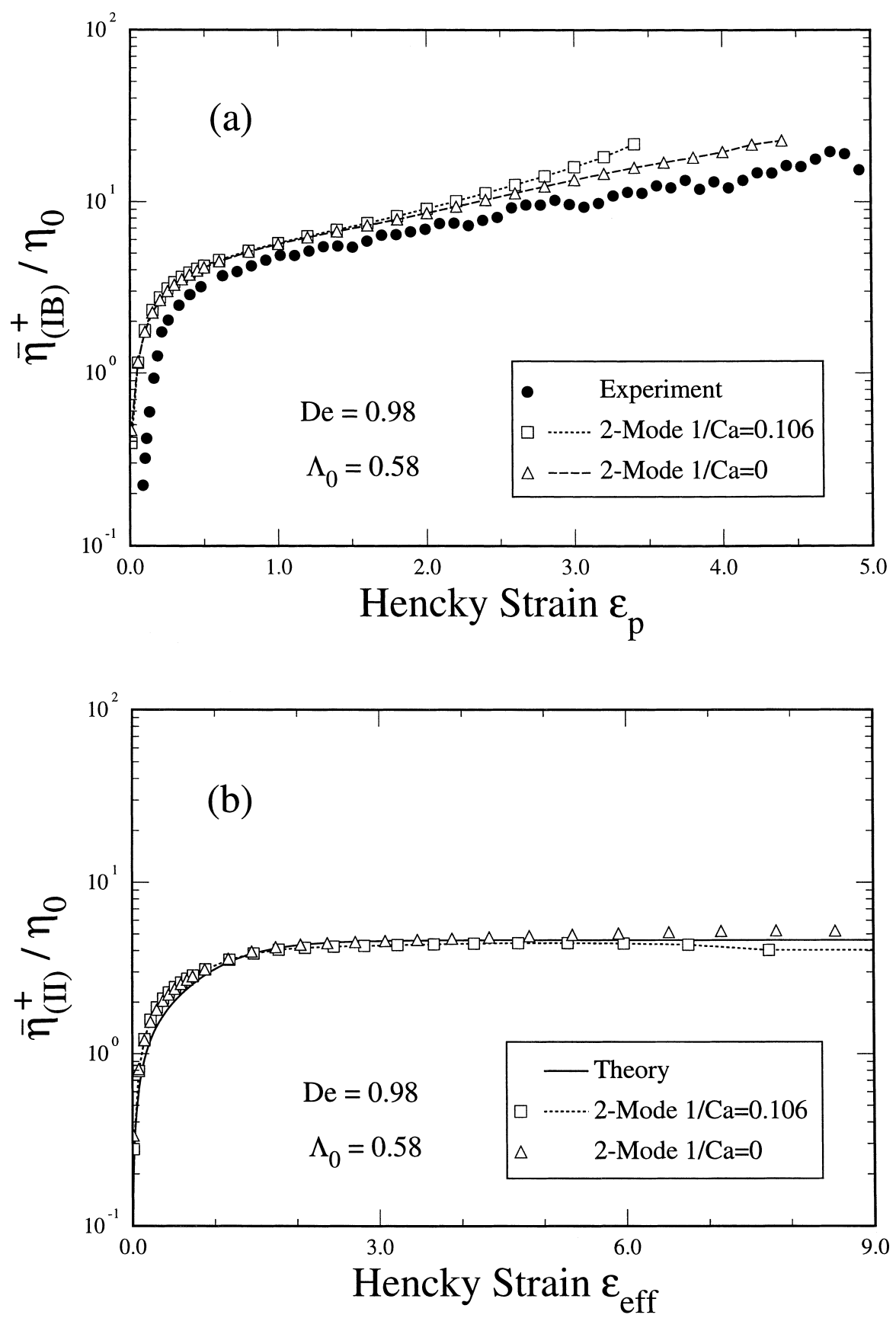

Fig. 8. Test run 1: $\mathrm{De}=0.98$ and $\Lambda_{0}=0.58$. Comparison of the Trouton ratio simulated using a two-mode Giesekus model with the experimental data for a weakly strain-hardening fluid described in Table 1. (a) Comparison using the type IB analysis. The curve $1 / \mathrm{Ca}=0$ corresponds to a computation in which the surface tension along the free surface is neglected. (b) Comparison using the type II analysis. 
The major difference between the type IB and type II analysis is how the local extensional strain rate is approximated. In the type IB formulation, the imposed axial extension rate $\dot{E}$ is used to approximate the local extensional strain rate at the mid-plane. This is clearly a poor approximation, because $\dot{E}$ underestimates the local extensional strain rate $\dot{\epsilon}_{\text {eff }}$ at the mid-plane, especially at large strains. As a result, the Trouton ratio presented in Fig. 7(a) does not approach a steady state; instead, it increases monotonically with strain (time). In interpreting experimental results for filament stretching devices, it should be noted that, for weakly strain-hardening fluids, the type IB analysis provides an upper bound of the actual extensional viscosity since the actual deformation rate is always higher than the imposed axial deformation rate.

In Figs. 8 and 9, we present the experimental and numerical results for the first and second test runs with imposed extension rate $\dot{E}=2.32,3.42 \mathrm{~s}^{-1}$ and Deborah numbers $\mathrm{De}_{1}=0.98,1.44$, respectively. In Fig. 8(a) and Fig. 9, the measurements and numerical data are processed by using the type IB analysis and the transient extensional viscosity is plotted as a transient Trouton ratio, versus the axial Hencky strain $\epsilon_{\mathrm{p}}$. The calculations in Fig. 8(a) were performed both including surface tension (corresponding to $\left.\mathrm{Ca}^{-1}=0.106\right)$ and without $\left(\mathrm{Ca}^{-1}=0\right)$. Clearly the effects of surface tension are small and will become progressively less important at higher Deborah numbers as the tensile viscoelastic stresses in the fluid column become even larger. In Fig. 8(b) and Fig. 9, the results of numerical simulations are postprocessed using the type II analysis given in Eq. (21) and the predicted Trouton ratio, $\eta_{\mathrm{II}}^{+}$, is plotted as a function of the effective strain $\epsilon_{\text {eff }}$ accumulated at the mid-plane. As we can see, the local strain $\epsilon_{\text {eff }}$ near the mid-plane is much higher than the average strain $\epsilon_{\mathrm{p}}$. The 'theory' curves shown in Fig. 8(b) and Fig. 9 are again the theoretical prediction of the three-mode Giesekus model for ideal uniaxial

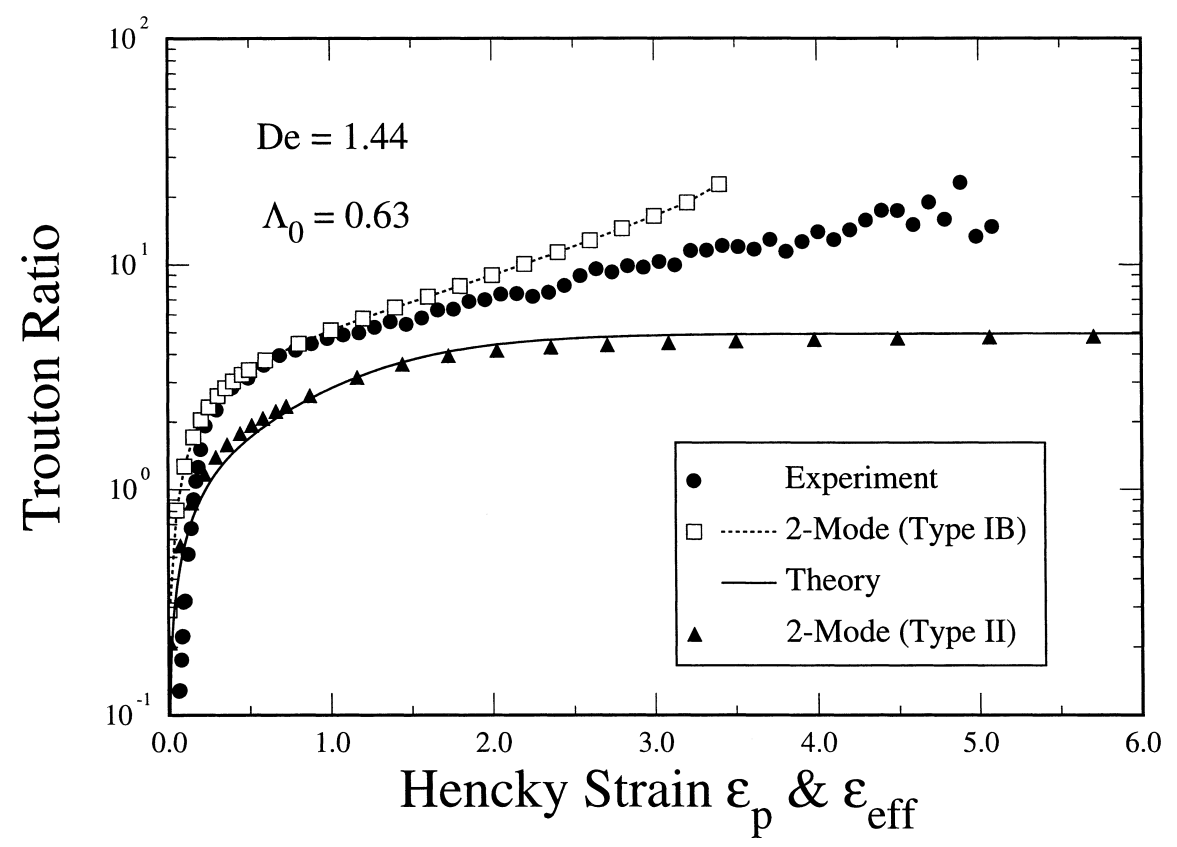

Fig. 9. Comparison of the Trouton ratio simulated using two-mode Giesekus model with the experimental data for the test run 2 described in Table $2\left(\mathrm{De}=1.44\right.$ and $\Lambda_{0}=0.63$ ). 
elongational flow. The use of $\dot{\epsilon}_{\text {eff }}$ in the type II analysis provides a far superior approximation of the local extensional strain rate at the mid-plane. As a result, the numerical predictions shown in Fig. 8(b) and Fig. 9 approach steady-state values that agree well with theoretical results. In comparing Figs. 7-9, it should be noted that for data points computed using the type IB analysis, the relevant strain measure for the abscissa is the total axial strain $\epsilon_{\mathrm{p}}=\dot{E} t$, whereas for the data computed using the type II analysis the appropriate measure is the effective strain $\epsilon_{\mathrm{eff}}$ experienced by fluid elements near the mid-plane which can be computed using Eq. (18).

\section{Stress relaxation}

In this section, we study relaxation of the tensile stresses in the weakly strain-hardening test fluid. We consider the two-stage extensional deformation history in which the filament is first stretched at an exponential velocity prescribed by Eq. (1) up to a pre-set strain, then the stretching is stopped and the relaxation in tensile stresses and the necking of the filament begin. The free surface evolution and the fluid kinematics during relaxation are studied via full-scale numerical simulations and a 1-D asymptotic analysis. The gravitational body force is neglected in the simulations.

Following the convention in the literature, the transient extensional viscosity and the transient Trouton ratio in relaxation are defined as

$$
\operatorname{Tr}^{-} \equiv \bar{\eta}^{-}\left(\dot{E}, t, t_{0}\right)=\frac{\tau_{z z}-\tau_{r r}}{\dot{\epsilon}_{0}}
$$

where $t_{0}$ is the time when the moving end-plate starts to decelerate and $\dot{\epsilon}_{0}$ is a dimensionless measure of the extensional strain rate at the moment $t_{0}$ (scaled by $1 / \dot{E}$ ). In this work we use the effective extension rate at the mid-plane as the appropriate scale for $\dot{\epsilon}_{0}$. For consistency with previous work [16], the characteristic time scale chosen for studies of stress relaxation is taken to be $\dot{E}^{-1}$ and we define a 'generalized Hencky strain', $\epsilon_{\mathrm{g}}$. This dimensionless time is identical to the average Hencky strain measure used during elongation and, after stretching is stopped at a final strain of $\epsilon_{\mathrm{p}}=\dot{E}\left(t_{0}+\delta t_{\mathrm{r}}\right)$, is given by

$$
\epsilon_{\mathrm{g}}=\epsilon_{\mathrm{p}}+\dot{E}\left(t-t_{0}-\delta t_{\mathrm{r}}\right)=\dot{E} t
$$

where $\delta t_{\mathrm{r}}$ is the ramping time. For the sudden stop, we have $\delta t_{\mathrm{r}}=0$. When the ramping time is non-zero, the motion of the end-plate is complete at $t=t_{0}+\delta t_{\mathrm{r}}$.

Of course, for a relaxing viscoelastic filament, a more appropriate characteristic time scale would be the relaxation time $\lambda_{1}$ and, for a Newtonian filament undergoing capillary thinning, the only relevant time scale in the problem following cessation of stretching is $\mu R_{0} / \sigma$, however, the present choice permits the evolution of both viscoelastic and Newtonian filaments during and after cessation of stretching to be conveniently represented on the same graph.

We first consider a typical case of stress relaxation for the test fluid after stretching at $\mathrm{De}=2.0$. In this case, the stretching is stopped at a strain of $\epsilon_{\mathrm{p}}=3.0$ with zero ramping time. For simplicity, the problem is simulated using the one-mode Giesekus model. The corresponding numerical solution for a Newtonian fluid is also computed to serve as a reference state. The simulated free surface profiles at six selected strains are presented in Fig. 10. Due to the assumed symmetry with respect to the mid-plane between the two end-plates, only the top half of the deforming filament is shown. 


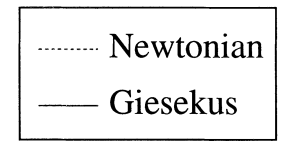

$\mathrm{De}=2.0$

$\Lambda_{0}=0.54$

$\mathrm{Ca}^{-1}=0.055$

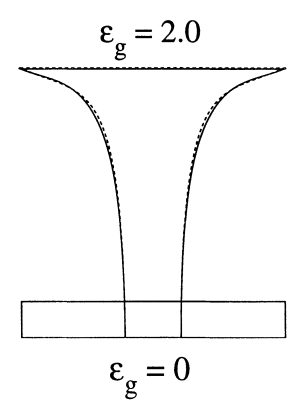

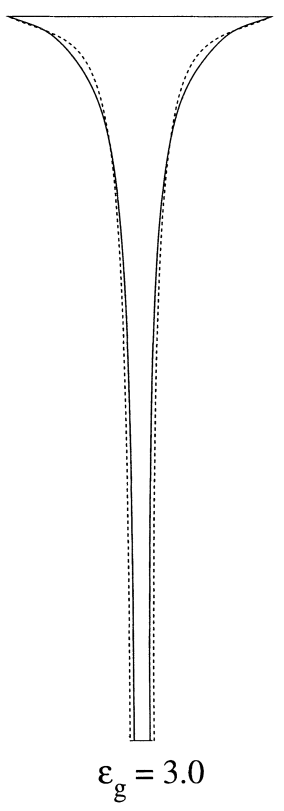
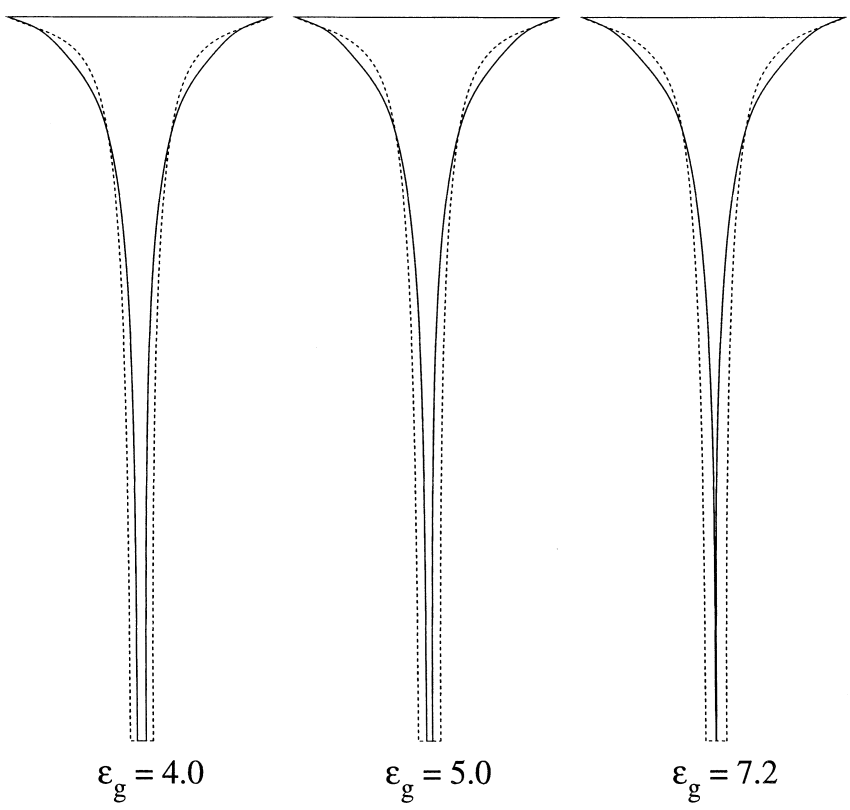

Fig. 10. Evolution of free surface profile and necking failure during a simulated stress relaxation test. The filament is stretched to $\epsilon_{\mathrm{g}}=3.0$, then the stretching is suddenly stopped. The weakly strain-hardening fluid is simulated by a one-mode Giesekus model. The generalized Hencky strain $\epsilon_{\mathrm{g}}$ is calculated using Eq. (23).

As we can see from Fig. 10, the difference in free surface shape between the two fluids is very small at $\epsilon_{\mathrm{g}}=2.0$. After stretching to $\epsilon_{\mathrm{g}}=3.0$, the central portion of the viscoelastic fluid column becomes slightly thinner than that of the Newtonian fluid. During the relaxation stage, the gradual thinning in the Newtonian filament radius is driven by surface tension and, for the selected fluid parameters, the change in free surface shape is very slow, as shown by the profiles at $\epsilon_{\mathrm{g}}=4.0,5.0$ and 7.2 in Fig. 10. However, the necking behavior of the weakly strain-hardening test fluid predicted by the Giesekus model is dramatically different from the Newtonian fluid. It can be seen from the simulated free surface profiles at $\epsilon_{\mathrm{g}}>3.0$ that the necking rate of the test fluid is much faster than the Newtonian case.

In Fig. 11, we present a more quantitative description of the simulated fluid kinematics as characterized by the mid-plane radius and the effective extension rate. For the weakly strain-hardening test fluid, the initial radial deformation is very similar to the Newtonian filament within the small strain range $\epsilon_{\mathrm{g}} \leq 2.0$. As the strain increases, the necking in the fluid filament starts to accelerate. At $\epsilon_{\mathrm{g}}=3.0$, the necking rate of the viscoelastic test fluid is about twice that in the Newtonian fluid. For the Newtonian fluid, the two-stage deformation history can be easily recognized from the sudden change in the slope of $R_{\text {mid }}(t)$ curve shown in Fig. 11(a) or from the discontinuity in the magnitude of the local extension rate shown by the $\dot{\epsilon}_{\text {eff }}$ curve in Fig. 11(b). Also shown in Fig. 11 are the predictions of a 1-D theory for the filament breakup which we now proceed to describe briefly.

Previous work by Bousfield et al. [22] has shown how a set of 1-D 'thin filament' equations can be used to model the evolution of a viscoelastic jet from an initial perturbed configuration. This 1-D theory accurately captures both the linear viscoelastic amplification in the initial disturbance growth rate and the subsequent non-linear stabilization computed in more expensive finite element calculations. 

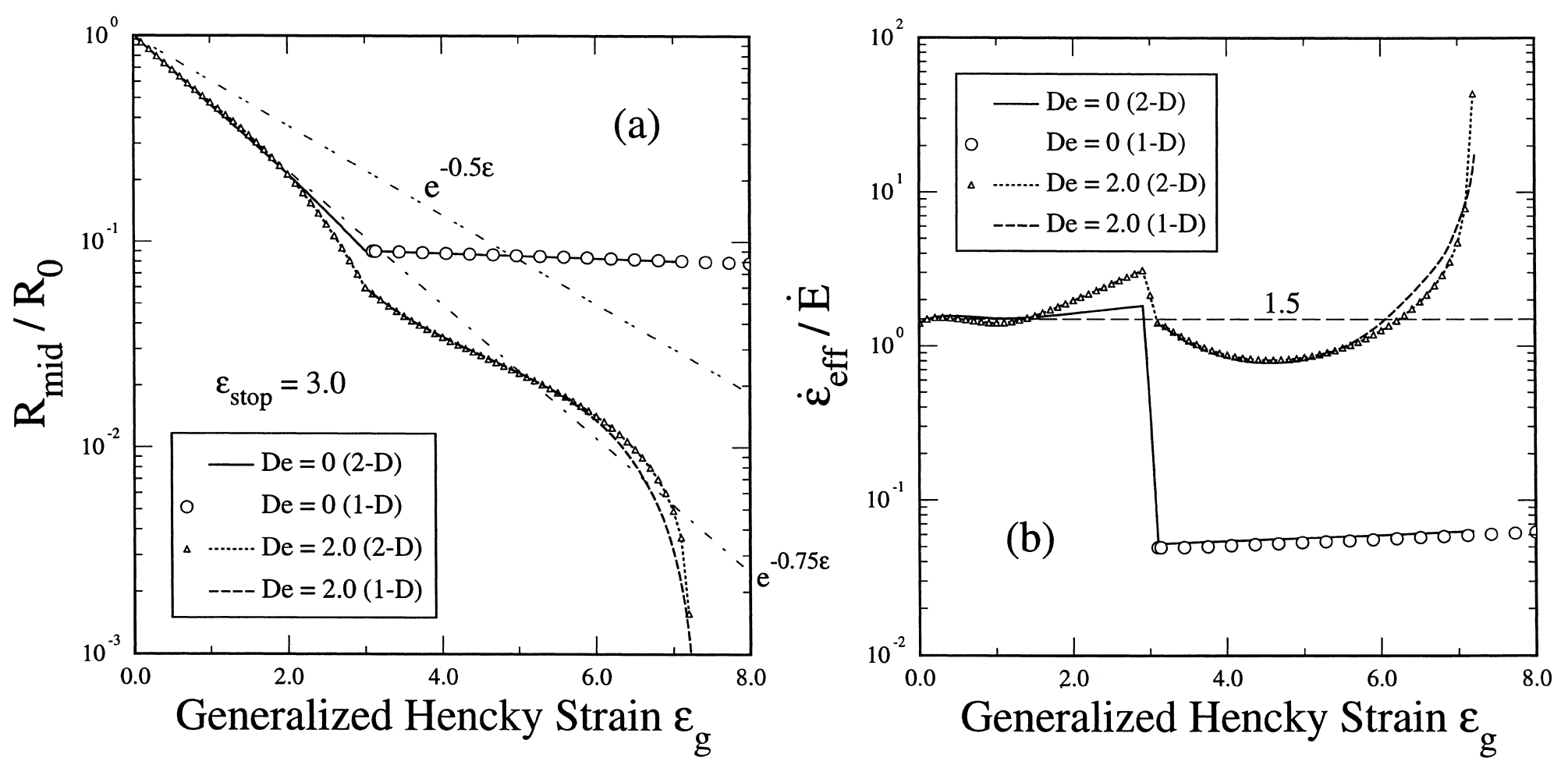

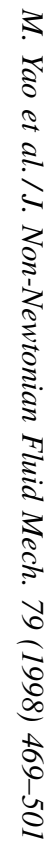

Fig. 11. A quantitative comparison of the flow kinematics during the stretching and subsequent relaxation process. $\mathrm{De}=0$ is the Newtonian case, and $\mathrm{De}=2.0$ corresponds to the weakly strain-hardening test fluid. The two sets of results for each liquid are obtained by full-scale 2-D simulation (POLYFLOW) and a 1-D asymptotic solution (1-D). (a) The mid-plane radius $R_{\text {mid }}$ as a function of the generalized Hencky strain $\epsilon_{\mathrm{g}}$ defined in Eq. (23); (b) the effective extension rate $\dot{\epsilon}_{\text {eff }}$ defined in Eq. (17). 
Asymptotic analysis of the 1-D equation set by Renardy [20,21] has further shown that the dynamical evolution of the necked jet is a sensitive function of the constitutive model chosen to describe the viscoelastic fluid. We have adapted these ideas to explore the role of extensional fluid rheology on the evolution of the long slender fluid column generated in a filament stretching apparatus following cessation of elongation.

A common difficulty encountered with 1-D approximations for viscoelastic flows is the choice of appropriate initial conditions. The analyses of viscoelastic jets [20-22] have shown that the dynamical evolution in the jet profile at short and intermediate times is modified appreciably by the existence of an initial tensile stress difference in the fluid column. As Hinch and Entov [40] remark, this sensitivity to the (unknown) initial conditions makes it difficult to use measurements of filament breakup to quantitatively ascertain values of viscoelastic constitutive parameters. In the present work we seek to investigate the unstable necking of a viscoelastic filament that develops during the process of stress relaxation following cessation of a strong uniaxial elongation. As a result of the previous deformation there is a significant initial tensile stress difference in the column; however, one of the principal benefits of a filament stretching device is that the same Lagrangian fluid elements are followed from their initial configuration as a uniform, fully-relaxed cylindrical liquid bridge through a strong uniaxial deformation and into the ultimate stress-relaxation/filament break-up regime. Our comparisons of the numerical simulations and experimental observations during stretching have shown that we can quantitatively predict the tensile stress growth in the elongating filament. We can thus use the computed spatial profile of the polymeric stresses at the cessation of stretching as the initial condition for integration of the set of 1-D equations of motion governing the evolution of a slender fluid filament.

These governing equations are formulated in a convenient Lagrangian form by Renardy [20,21]. In this representation the axial location $z(t)$ of fluid elements with initial location $Z_{0}$ ( at time $t_{0}$ ) is followed as a function of time. The deformation in the fluid column is represented by the dimensionless stretch $S\left(Z_{0}, t\right)$ of each element which is defined in a $1-\mathrm{D}$ deformation by

$$
\left(\frac{\partial z}{\partial Z_{0}}\right)_{t} \equiv S\left(Z_{0}, t\right)=\frac{R_{0}^{2}}{R\left(Z_{0}, t\right)^{2}}
$$

Here time and position are non-dimensionalized as described in Eq. (2), and the initial Lagrangian domain spans $0 \leq Z_{0} \leq L(t) / R_{0}$. For a 1-D flow, in which the axial velocity in the column is only a function of $z$, the deformation rate at each axial position of the filament is given by

$$
\dot{\epsilon} \equiv D_{z z}\left(Z_{0}, t\right)=\frac{1}{S}\left(\frac{\partial S}{\partial t}\right)_{Z_{0}}
$$

The governing equations in dimensionless form are then

$$
\begin{aligned}
& 3 \beta_{\mathrm{s}} \frac{\partial S}{\partial t}=S^{2} f(t)-S\left(T_{z z}-T_{r r}\right)-\mathrm{Ca}^{-1} S^{3 / 2} \\
& \operatorname{De} \frac{\partial T_{z z}}{\partial t}=T_{z z}\left[2 \operatorname{De} \dot{\epsilon}-1-\frac{\operatorname{De} \alpha T_{z z}}{1-\beta_{\mathrm{s}}}\right]+2\left(1-\beta_{\mathrm{s}}\right) \dot{\epsilon} \\
& \operatorname{De} \frac{\partial T_{r r}}{\partial t}=-T_{r r}\left[\operatorname{De} \dot{\epsilon}+1+\frac{\operatorname{De} \alpha T_{r r}}{1-\beta_{\mathrm{s}}}\right]-\left(1-\beta_{\mathrm{s}}\right) \dot{\epsilon}
\end{aligned}
$$


Here we have used the same dimensional scalings defined in Section 3.2 (Eq. (2)), $T_{z z}$ and $T_{r r}$ are the 1-D approximations of the polymeric contributions to the total stress, and $\mathrm{Ca}$ is the capillary number defined in Eq. (3). The dimensionless tensile force $f(t)$, is scaled with the initial conditions and is uniform along the filament in Eq. (26a) but decays with time. Since the total length of the column is not changing with time, the force can be found from an integral constraint [21] as

$$
f \equiv \frac{F_{z}}{\pi R_{0}^{2} \eta_{0} \dot{E}}=\frac{\int_{0}^{\Lambda\left(t_{0}\right)}\left[S\left(T_{z z}-T_{r r}\right)+\mathrm{Ca}^{-1} S^{3 / 2}\right] \mathrm{d} Z_{0}}{\int_{0}^{\Lambda\left(t_{0}\right)} S^{2} \mathrm{~d} Z_{0}}
$$

Given a filament profile $R\left(Z_{0}, t_{0}\right)$, and an initial distribution of the polymeric stresses $T_{z z}\left(Z_{0}, t_{0}\right)$ and $T_{r r}\left(Z_{0}, t_{0}\right)$, Eqs. (26a),(26b),(26c) and (27) can be integrated in time to compute the evolution in the filament profile. If the filament breaks in finite time then the Lagrangian stretch of the material element at the mid-point diverges at a critical time $t_{\mathrm{c}}$ as $S\left(z_{0}=0, t\right) \sim\left(t_{\mathrm{c}}-t\right)^{-b}$. Renardy [20,21] shows that for the Giesekus model the critical exponent is $b=2$ corresponding to a linear decrease in the mid-point radius $R_{\text {mid }} / R_{0}$ at long times.

The predictions of the one-dimensional theory are compared with finite element calculations in Fig. 11. Clearly, the 1-D set of equations provides a good description of the evolution in the filament profile for both the Newtonian and viscoelastic fluids. For the Newtonian filament, capillary pressure in the necked region drives the progressive decrease in the filament radius; however, for the high fluid viscosity selected $\left(\mu=\eta_{0}=34.95 \mathrm{Pas}\right)$, the characteristic time scale for breakup is very long $\left(t_{\mathrm{c}} \sim \mu R_{0} / \sigma=4.08 \mathrm{~s}\right)$. Little change in the filament profile is observed on the scale of Fig. 11 $\left(\Delta t=\Delta \epsilon_{\mathrm{g}} / \dot{E} \approx 1.07 \mathrm{~s}\right)$, although close inspection shows that the mid-point radius does decrease monotionically and the extension rate increases slowly. By contrast, the viscoelastic filament undergoes an increasingly rapid rate of necking and the extension rate increases with time. Analysis of the 1-D curve using the method proposed by Renardy [21] shows that the critical (dimensionless) time for breakup is $t_{\mathrm{c}}=7.36$ and the exponent $b=2.0$ in agreement with asymptotic predictions. The slight deviations between the 1-D equations and the full two-dimensional finite-element computations arise from the assumed form of the 1-D kinematics which cannot capture the no-slip boundary conditions imposed by the end-plates of the device. In this region, detailed analysis of the finite element simulations (not shown here) indicates that the flow is locally two-dimensional and, furthermore, the axial curvature of the filament surface is no longer small.

To further investigate why the necking behavior of the weakly strain-hardening test fluid differs so significantly from the Newtonian fluid, we examine the spatial variation of the extensional strain rate within the liquid filament. Fig. 12 shows the axial component of the rate-of-deformation tensor, $D_{z z}$, along the centerline of the filament $(r=0)$ at three selected strain levels. The plot at $\epsilon_{\mathrm{g}}=3.0^{-}$ (immediately before stretching ceased) shows a small region near the moving end-plate $0.35<z \leq 0.5$ where $D_{z z}<0$. In this region the polymeric liquid undergoes elastic recoil. However, following removal of the imposed external axial deformation, the profile at $\epsilon_{\mathrm{g}}=3.1$ shows that there is a sudden increase in the strength and spatial extent of the viscoelastic recoil near the end-plate. Very close to the axial end-plate the elastic stresses relax when the external deformation is removed and the filament expands slightly in the radial direction as fluid elements recoil. Consequently the filament radius decreases near the mid-plane and the rate of necking in this region increases. The region of elastic recoil (i.e. $D_{z z}<0$ ) is thus progressively localized at long times and the deformation rates in the necking column slowly intensify in magnitude and move away from the rigid end-plates. Also shown in 

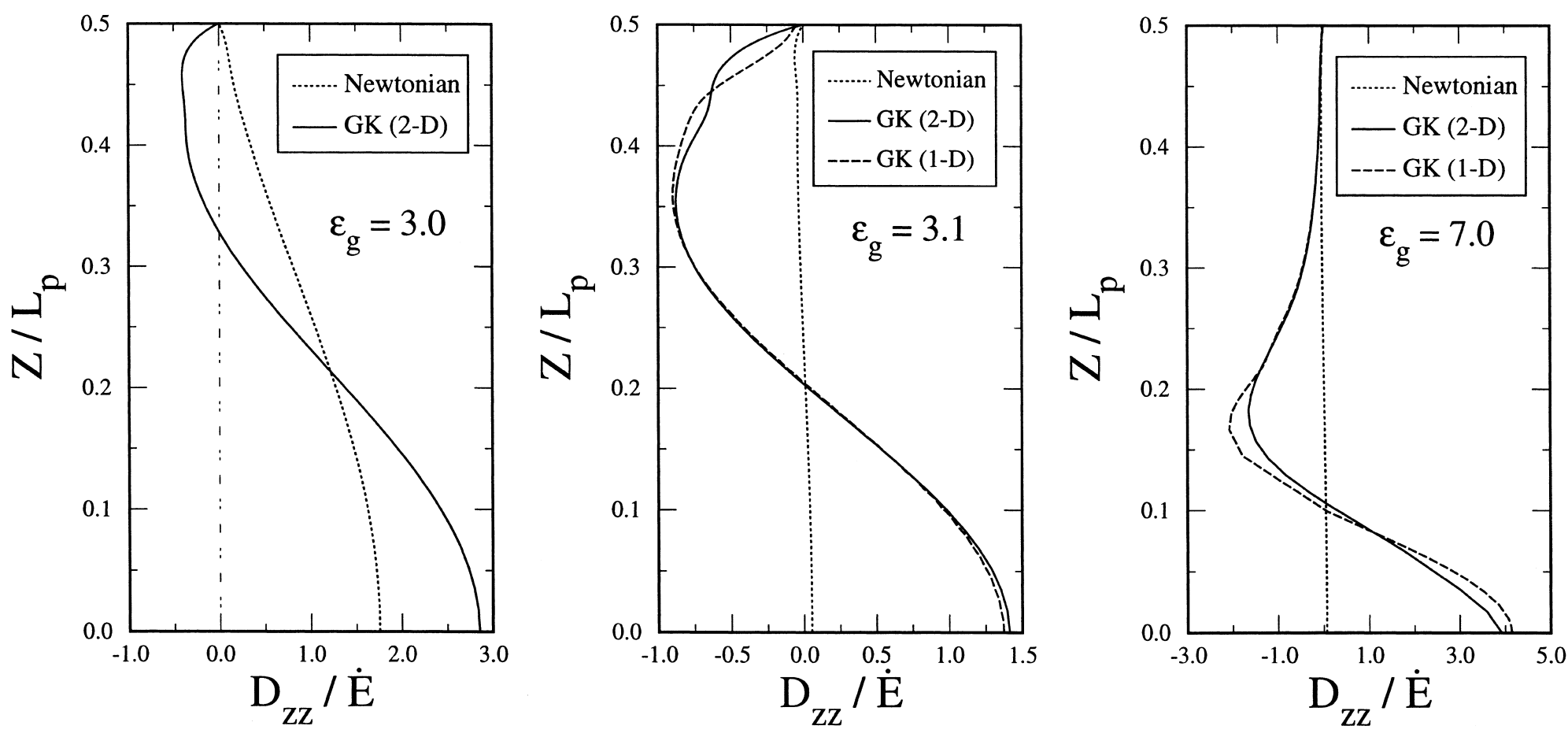

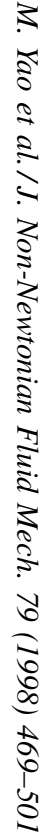

Fig. 12. Spatial variation of the axial component of the rate-of-deformation tensor $D_{z z}$ along the centerline for a Newtonian fluid and for the Gresekus model (GK) during the stretching (at $\epsilon_{\mathrm{g}}=3.0^{-}$immediately before stretching is stopped) and in relaxation (at $\epsilon_{\mathrm{g}}=3.1$ and 7.0 ). $z=0$ is the mid-plane and $z / L_{\mathrm{p}}=0.5$ is the top end-plate. 


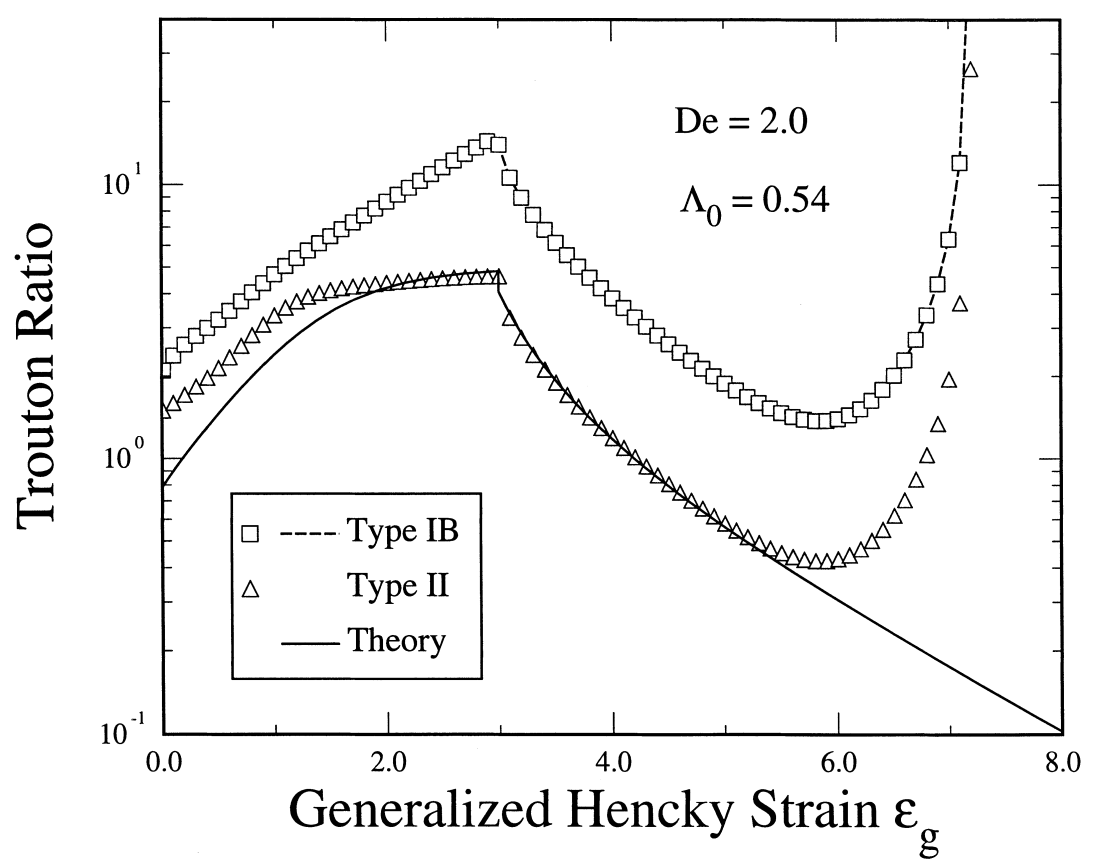

Fig. 13. The non-linear stress growth and relaxation for the weakly strain-hardening fluid simulated by the one-mode Giesekus model. The stretching rate is $\dot{E}=4.68 \mathrm{~s}^{-1}$ and stretching is suddenly stopped at $\epsilon_{\mathrm{g}}=3.0$. The Trouton ratio is calculated by both the type IB (20b) and type II (22) analyses. The 'theory' curve is obtained assuming homogeneous fluid kinematics for an ideal uniaxial elongational flow.

Fig. 12(b-c) are the predictions of the 1-D thin filament equations. This asymptotic theory accurately predicts the kinematics inside the necking fluid column except in small regions near the end-plate where the full numerical calculations show that the fluid motion is weakly two-dimensional.

Measurements of the decaying tensile force have been used to investigate stress relaxation following cessation of elongational flow in strongly strain-hardening dilute polymer solutions $[7,10,16,38]$. In Fig. 13 we show the computed evolution in the Trouton ration $\mathrm{Tr}^{-}$for the one-mode Giesekus model, and a comparison with the theoretical prediction for ideal uniaxial elongational flow. When the Trouton ratio is computed using the type IB formulation and plotted as a function of $\epsilon_{\mathrm{g}}$ (as shown by the squares in Fig. 13) the predicted stress growth during stretching is larger than expected theoretically due to the non-homogeneity in the local extensional strain rate near the mid-plane. Consequently, if the imposed extension rate $\dot{E}$ is used as the appropriate measure of deformation rate in the Trouton ratio $\mathrm{Tr}^{-}$defined in Eq. (22) then the computed results do not agree with theoretical expectation. However, if we compute $\operatorname{Tr}^{-}$with the actual deformation rate $\dot{\epsilon}_{\text {eff }}$ at the instant that deformation is creased, then we can see from Fig. 13 that the stress relaxation in the filament is initially as expected theoretically. However, after a generalized strain of $\Delta \epsilon_{\mathrm{g}} \approx 2$ corresponding to an elapsed time of order one fluid relaxation time $\left(\Delta t / \lambda_{1} \equiv \Delta \epsilon_{\mathrm{g}} / \mathrm{De} \approx 1.0\right)$, the polymeric stress difference passes through a minimum and begins to increase rapidly due to the unstable necking in the filament. It is important to note that although the stress in the filament diverges (as does the local deformation rate), the force $f(t)$ in the filament computed using either the 1-D filament equations or the full two-dimensional finite element simulation 


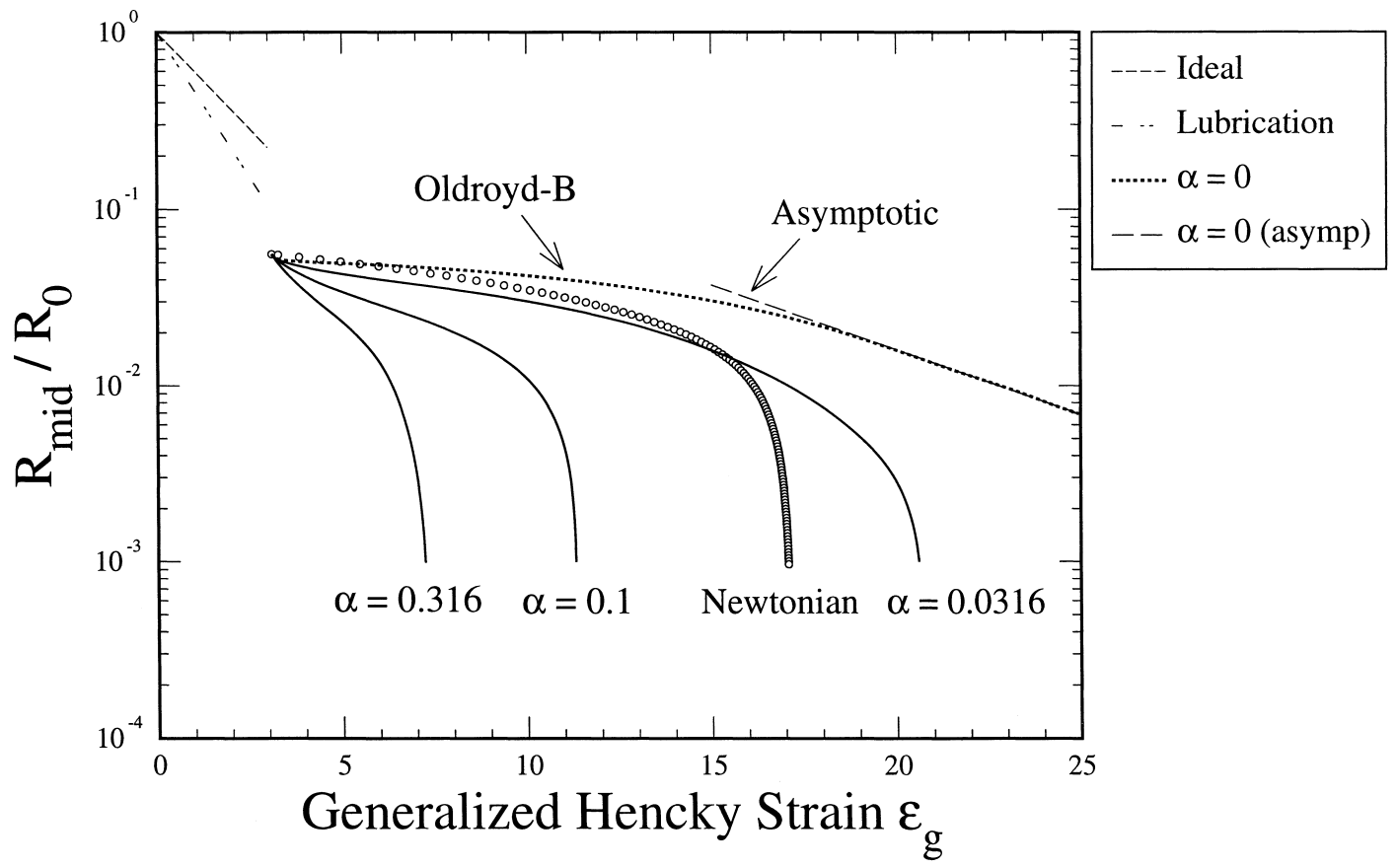

Fig. 14. Influence of extensional fluid rheology on the necking failure of a viscoelastic fluid thread in a filament stretching device. The initial conditions at $\epsilon_{\mathrm{g}}=3.1$ are taken from the numerical simulation shown in Figs. 10 and 11 . As the Giesekus parameter $\alpha$ is decreased, the 1-D theory (Eqs. (26)-(27)) predicts that the time to breakup increases monotonically. In the Oldroyd-B limit $(\alpha=0)$ the filament radius decreases exponentially in agreement with asymptotic predictions and the thread does not break in finite time. For comparison, the capillary-driven breakup of a corresponding viscous Newtonian filament is shown by the hollow symbols.

decreases monotonically. The results in Fig. 13 suggest that filament stretching devices can be used successfully to monitor the initial stages $\left(\Delta t \sim \lambda_{1}\right)$ of extensional stress relaxation even in weakly strain-hardening materials such as the concentrated polystyrene solution. Of course, in the experiments, additional factors such as the finite dynamic range of the force transducer and gravitational sagging of the column may also be important at long times.

Two recent numerical studies $[19,25]$ have shown that the dynamical evolution of viscoelastic filaments and the predicted rate of necking failure are very sensitive to the magnitude and type of nonlinearity governing the dynamics of the tensile stress growth during uniaxial elongation. This variation in the level of strain-hardening in the fluid also dramatically influences the unstable necking of the filament following cessation of stretching as we show in Fig. 14. Here we investigate the effect of decreasing the parameter $\alpha$ in the Giesekus model while holding all other variables (e.g. surface tension and viscosity) constant. In order to eliminate the variation in initial conditions that would arise from performing a full numerical simulation of the initial stretching deformation to a fixed Hencky strain $\epsilon_{\mathrm{p}}$ using different sets of constitutive parameters, we use the 1-D model equations presented above (Eqs. $(26 a-c)$ and (27)) and take the initial stress distribution to be that computed numerically for deformation of a fluid sample described by a single-mode Giesekus model with $\alpha=0.316$ to a final strain $\epsilon_{\mathrm{p}}=3.0$ (i.e. the conditions shown in Figs. 10 and 11). Initially, there is a relaxation of the 
polymeric stresses in the filament of the form shown in Fig. 13 and consequently little fluid motion; however, at longer times the deformation rate begins to climb and the rate of necking increases. As $\alpha$ is decreased the onset of filament failure is delayed to progressively longer times, but for each value of $\alpha \neq 0$, asymptotic analysis of the stretch at the mid-plane $S\left(Z_{0}=0, t\right)$ shows that the critical exponent is $b=2$ corresponding to a linear decrease in the filament mid-point radius near breakup. By contrast, for the Oldroyd-B model $(\alpha=0)$, the simulations show that at long times the filament radius decreases not linearly but exponentially and the deformation rate approaches a constant value (although as noted by Renardy [21], the numerical solution in fact becomes oscillatory at very long times). Asymptotic analyses presented first by Bousfield et al. [22] and later by Hinch and Entov [40] show that for the Oldroyd-B fluid, the mid-point radius should decrease as $R_{\text {mid }} / R_{0} \sim \exp \left[-t /\left(3 \lambda_{1}\right)\right]=\exp \left[-\epsilon_{\mathrm{g}} /(3 \mathrm{De})\right]$ at long time as indicated in Fig. 14. Also shown in Fig. 14 by the symbols is the evolution of a Newtonian filament with same initial configuration but with $T_{z z}=T_{r r}=0$ and $\lambda_{1}=0$. In this case the filament fails solely due to capillary-driven thinning. At short times following cessation of stretching, close inspection of Fig. 14 shows that the necking of all the viscoelastic filaments is more rapid than the corresponding Newtonian filament as predicted by linear stability theory [73]. However, at longer times, the column can be partially or completely stabilized against capillary breakup. The extent of this stabilization is clearly dependent on the magnitude of the strain-hardening predicted by the constitutive model.

\section{Conclusions}

We have presented numerical simulations using the finite element package POLYFLOW developed by Crochet and co-workers to investigate the dynamical evolution of viscoelastic fluid columns in filament stretching devices during elongation and subsequent stress relaxation. A detailed comparison of computations with single- and multi-mode formulations of the Giesekus model has been presented to examine differences in the predicted viscoelastic behavior resulting from the relaxation spectrum and the constitutive non-linearities characterizing the fluid.

Comparisons show that the multi-mode calculation provides a much better prediction for the initial stress growth in the material at short times and small Hencky strains, and compares very favorably to both experimental measurement and theoretical prediction. At larger strains, simulations using the multi-mode constitutive model predict a significantly stronger elastic recoil of the fluid near the endplate. As a result, the multi-mode solution has a higher local extension rate near the mid-plane, which leads to a more rapid necking in the column during stretching. Although the numerical calculations cannot be carried all the way to ultimate filament rupture (due to the very large element deformations that develop at large strains) the rate of necking increases without bound and the filament is clearly destabilized with respect to the identical elongation of a Newtonian liquid bridge, despite the strainhardening in the extensional viscosity. This enhancement in the unstable necking is not dependent on pressure gradients arising from surface tension but arises purely from viscoelastic effects such as elastic recoil of the fluid elements near the end-plates. Improved resolution of the fluid's viscoelastic properties via incorporation of a discrete spectrum of relaxation times increases the rate of necking, and analyses based on the single-mode calculations can be considered as more conservative estimates of the time to break-up. Comparison with theoretical predictions for ideal homogeneous uniaxial elongation shows that, even for weakly strain-hardening fluids, it is possible to make quantitative measurements of 
the transient uniaxial elongational viscosity if, in addition to measurement of the tensile force in the column, it is possible to obtain an accurate determination of the local rate of deformation at the midplane.

The differences in filament evolution resulting from variations in the extensional rheology of the test fluid are even more evident in our numerical studies of the 'stress relaxation experiments' commonly performed in filament stretching devices following cessation of elongation. For a viscous Newtonian fluid filament, a sudden cessation of the extensional flow can be approximately achieved after a sudden stop of the end-plate motion and, in the absence of gravity, the ensuing evolution of the initially necked configuration is driven only by capillary pressure. Although the filament will eventually fail in a finite time, the necking rate at the mid-plane is initially very slow due to the high viscosity of the test fluids commonly used in filament stretching devices. The dynamical evolution of a viscoelastic test fluid after cessation of stretching differs dramatically from the Newtonian fluid counterpart. The filament may be stabilized or destabilized (i.e. the time to breakup can be increased or reduced, respectively, as shown in Fig. 14) depending on the magnitude and rate of extensional strain-hardening predicted by the model. Depending on the choice of non-linear constitutive parameters, up to three characteristic phases in the filament evolution can be identified following cessation of stretching. These regions can be distinguished by the magnitude of the axial deformation rate $\dot{\epsilon}_{\text {eff }}$ at the filament mid-plane which characterizes the rate of necking in the fluid column. Initially there is a sudden, large decrease in the rate of necking after the external forcing is removed, and the viscoelastic stress difference in the elongated fluid column relaxes almost as predicted for cessation of ideal uniaxial extension. This relaxation in the axial stress is accompanied, however, by elastic recoil of the fluid elements near the rigid end-plates and a slow decrease in the diameter of the necked region near the filament mid-plane. Although this rate of necking is small at first, it is always larger than that observed in the corresponding Newtonian filament, in agreement with linear viscoelastic stability theory [73]. At longer times, strainhardening in the necking region may become significant enough (depending on the values of the nonlinear constitutive parameter characterizing the extensional fluid rheology), so that the local effective extension rate at the mid-plane of the filament falls below that of the corresponding Newtonian filament, and the fluid column is therefore (partially) stabilized. For an Oldroyd-B fluid, which permits unbounded tensile stress growth, this is the terminal regime and asymptotic analysis shows that the final filament profile consists of a long thin fibril of highly stretched fluid connecting two quasi-static, fullyrelaxed fluid reservoirs (i.e. the 'beads-on-a-string' configuration [20-22]). However, if the extensional viscosity is bounded, then as the molecules in the fluid element at the mid-plane approach full extension, the filament enters the final stage of the filament breakup process. In this phase the radius decreases rapidly and the deformation rate in the filament starts to climb again. For the Giesekus model at least, the Lagrangian stretch diverges as $\left(t_{\mathrm{c}}-t\right)^{-2}$ and the mid-plane radius decreases linearly to zero at a critical time $t_{\mathrm{c}}$, as expected for a viscous Newtonian fluid [21]. These numerical observations demonstrate the key role of the transient extensional rheology of the fluid in governing the evolution of the fluid filament and are also self-consistent with the Considère criterion which is valid during the imposed stretching phase of the experiment and discussed in detail elsewhere [19,25].

Of course fluid inertia may modify the asymptotic evolution in the neck at the very final stages of breakup, and Entov and Hinch [40] have shown how a spectrum of relaxation times can change the viscoelastic dynamics at intermediate times. However, it is clear that if the initial radial profile $R\left(z, t_{0}\right)$ and tensile force $f\left(t_{0}\right)$ in the column are known, then careful measurements of the evolution in the midplane radius $R_{\text {mid }}(t)$ using a filament stretching device or a microfilament rheometer $[39,40]$ can be 
combined with either two-dimensional, time-dependent, finite element calculations or a 1-D slenderfilament theory to provide a sensitive probe of the transient extensional rheology of mobile viscoelastic fluids such as dilute and concentrated polymer solutions.

\section{Acknowledgements}

This work was supported by NASA's Microgravity Science and Application Program. Computer system and graphics supports from David A. Thompson at the Computational Microgravity Lab, NASA Lewis Research Center is acknowledged. It is a pleasure to acknowledge extended discussions with O. Hassager on the subject of necking in viscoelastic filaments.

\section{References}

[1] M.M. Denn, in: J.L. Lumley, A. Acrivos, L.G. Leal, S. Leibovich (Eds.), Research Trends in Fluid Dynamics, AIP Press, New York, 1996, pp. 69-76.

[2] J.E. Matta, R.P. Tytus, J. Non-Newtonian Fluid Mech. 35 (1990) 215-229.

[3] T. Sridhar, V. Tirtaatmadja, D.A. Nguyen, R.K. Gupta, J. Non-Newtonian Fluid Mech. 40 (1991) $271-280$.

[4] V. Tirtaatmadja, T. Sridhar, J. Rheol. 37 (1993) 1081-1102.

[5] S. Berg, R. Kröger, H.J. Rath, J. Non-Newtonian Fluid Mech. 55 (1994) 307-319.

[6] S.H. Spiegelberg, D.C. Ables, G.H. McKinley, J. Non-Newtonian Fluid Mech. 64 (1996) $229-267$.

[7] S.H. Spiegelberg, G.H. McKinley, J. Non-Newtonian Fluid Mech. 67 (1997) 49-76.

[8] M.J. Solomon, S.J. Muller, J. Rheol. 40 (1996) 837-856.

[9] A. Jain, D.S. Shackleford, K.W. Koelling, The 68th Society of Rheology Annual Meeting, 1997.

[10] J. van Nieuwkoop, M.M.O. Muller von Czernicki, J. Non-Newtonian Fluid Mech. 67 (1996) $105-123$.

[11] R.B. Bird, R.C. Armstrong, O. Hassager, Dynamics of polymeric liquids, vol. 1, Fluid Mechanics, 2nd ed., Wiley, New York, 1987.

[12] R.W.G. Shipman, M.M. Denn, R. Keunings, J. Non-Newtonian Fluid Mech. 40 (1991) 281-288.

[13] S. Gaudet, G.H. McKinley, H.A. Stone, Phys. Fluids 8 (1996) 2568-2579.

[14] M. Yao, G.H. McKinley, J. Non-Newtonian Fluid Mech. 74 (1998) 47-88.

[15] R. Sizaire, V. Legat, J. Non-Newtonian Fluid Mech. 71 (1997) 89-107.

[16] M.R.J. Verhoef, B.H.A.A. van den Brule, M.A. Hulsen, J. Non-Newtonian Fluid Mech., 1998, submitted.

[17] M.I. Kolte, H.K. Rasmussen, O. Hassager, Rheol. Acta 36 (1997) 285-302.

[18] P. Szabo, Rheol. Acta 36 (1997) 277-284.

[19] M. Yao, S.H. Spiegelberg, G.H. McKinley, J. Non-Newtonian Fluid Mech., 1998, submitted.

[20] M. Renardy, J. Non-Newtonian Fluid Mech. 51 (1994) 97-107.

[21] M. Renardy, J. Non-Newtonian Fluid Mech. 59 (1995) 267-282.

[22] D.W. Bousfield, R. Keunings, G. Marrucci, M.M. Denn, J. Non-Newtonian Fluid Mech. 21 (1986) $79-97$.

[23] J.M. Li, W. Burghardt, B. Yang, B. Khomami, J. Non-Newtonian Fluid Mech. 74 (1998) 151-194.

[24] Y.W. Ooi, T. Sridhar, J. Non-Newtonian Fluid Mech. 52 (1994) 153-162.

[25] O. Hassager, M.I. Kolte, M. Renardy, J. Non-Newtonian Fluid Mech. 76 (1998) 137-152.

[26] M. Considère, Annales des Ponts et Chaussées 9 (1885) 574.

[27] A.Y. Malkin, C.J.S. Petrie, J. Rheol. 41 (1997) 1-25.

[28] O. Hassager, personal communication, 1998.

[29] D.V. Boger, M.J. Crochet, R.A. Keiller, J. Non-Newtonian Fluid Mech. 44 (1992) 267-279.

[30] B. Purnode, M.J. Crochet, J. Non-Newtonian Fluid Mech. 65 (1996) 269-289.

[31] B. Purnode, M.J. Crochet, J. Non-Newtonian Fluid Mech. 77 (1998) 1-20.

[32] B. Debbaut, T. Avalosse, J. Dooley, K. Hughes, J. Non-Newtonian Fluid Mech. 69 (1997) $255-271$. 
[33] R. Sizaire, Ph.D. Thesis, Universite Catholique de Louvain, Louvain-la-Neuve, Belgium, Chap. 3 , 1998.

[34] R. Sizaire, V. Legat, J. Rheol., 1998, submitted.

[35] C. Bodart, M.J. Crochet, J. Non-Newtonian Fluid Mech. 54 (1994) 303-329.

[36] J.V. Satrape, M.J. Crochet, J. Non-Newtonian Fluid Mech. 55 (1994) 91-111.

[37] C. Beraudo, A. Fortin, T. Coupez, Y. Demay, B. Vergnes, J.F. Agassant, J. Non-Newtonian Fluid Mech. 75 (1998) 1-23.

[38] N. Orr, T. Sridhar, J. Non-Newtonian Fluid Mech. 67 (1996) 77-104.

[39] A.V. Bazilevsky, V.M. Entov, A.N. Rozhkov, in: D.R. Oliver (Ed.), Third European Rheology Conference, Elsevier, Amsterdam, 1990.

[40] V.M. Entov, E.J. Hinch, J. Non-Newtonian Fluid Mech. 72 (1997) 31-54.

[41] H. Giesekus, J. Non-Newtonian Fluid Mech. 11 (1982) 69-109.

[42] R.B. Bird, J.M. Wiest, J. Rheol. 29 (1985) 519-532.

[43] M.A. Hulsen, J. van der Zanden, J. Non-Newtonian Fluid Mech. 38 (1991) 183-222.

[44] D. Rajagopalan, J.A. Byars, R.C. Armstrong, R.A. Brown, J.S. Lee, G.G. Fuller, J. Rheol. 36 (1992) $1349-1375$.

[45] D. Rajagopalan, M.T. Arigo, G.H. McKinley, J. Non-Newtonian Fluid Mech. 65 (1996) 17-46.

[46] M.J. Crochet, A.R. Davies, K. Walters, Numerical Simulation of Non-Newtonian Flows, Elsevier, Amsterdam, 1984.

[47] M.J. Crochet, B. Debbaut, R. Keunings, J.M. Marchal, in: K.T. O’Brien (Ed.), Applications of CAE in Extrusion and Other Continuous Processes, Chap. 2, Carl Hanser, München, 1992.

[48] Polyflow User's Manual, ver. 3.5.0, Polyflow s.a., Place de 1'Université 16, B-1348 Louvainla-Neuve, Belgium, 1996.

[49] J.J. van Schaftingen, M.J. Crochet, Int. J. Numer. Methods in Fluids 4 (1984) 1065-1081.

[50] R. Keunings, in: C.L. Tucker III (Ed.), Fundamentals of Computer Modeling for Polymer Processing, Chap. 9, Carl Hanser, München, 1988.

[51] M.J. Crochet, Rubber Chem. Technol. 62 (1989) 425-455.

[52] F. Debae, V. Legat, M.J. Crochet, J. Rheol. 38 (1994) 421-442.

[53] M. Kawahara, N. Takeushi, Comput. Fluids 5 (1977) 33-45.

[54] M.J. Crochet, R. Keunings, J. Non-Newtonian Fluid Mech. 7 (1980) 199-212.

[55] M.J. Crochet, R. Keunings, J. Non-Newtonian Fluid Mech. 10 (1982) 85-94.

[56] M.J. Crochet, R. Keunings, J. Non-Newtonian Fluid Mech. 10 (1982) 339-356.

[57] R. Keunings, M.J. Crochet, M.M. Denn, Ind. Eng. Chem. Fundam. 22 (1983) 347-355.

[58] J.J. van Schaftingen, M.J. Crochet, J. Non-Newtonian Fluid Mech. 18 (1985) 335-351.

[59] B. Debbaut, M.J. Crochet, J. Non-Newtonian Fluid Mech. 20 (1986) 173-185.

[60] J.M. Marchal, M.J. Crochet, J. Non-Newtonian Fluid Mech. 26 (1987) 77-114.

[61] B. Debbaut, J.M. Marchal, M.J. Crochet, J. Non-Newtonian Fluid Mech. 29 (1988) 119-146.

[62] B. Debbaut, J. Non-Newtonian Fluid Mech. 36 (1990) 265-275.

[63] R. Keunings, J. Comput. Phys. 62 (1985) 199-220.

[64] R. Keunings, D.W. Bousfield, J. Non-Newtonian Fluid Mech. 22 (1987) 219-233.

[65] D.R. Lynch, W.G. Gray, J. Comput. Phys. 36 (1980) 135-154.

[66] S.L. Josse, B.A. Finlayson, J. Non-Newtonian Fluid Mech. 16 (1984) 13-36.

[67] D.W. Bousfield, R. Keunings, M.M. Denn, J. Non-Newtonian Fluid Mech. 27 (1988) 207-221.

[68] Ch. Bodart, M.J. Crochet, Theor. Comp. Fluid Dyn. 5 (1993) 57-75.

[69] G.C. Georgiou, M.J. Crochet, J. Rheol. 38 (1994) 1745-1755.

[70] P.M. Gresho, R.L. Lee, R.L. Sani, Recent Advances in Numerical Methods in Fluids, Pineridge, Swansea, 1980.

[71] P.M. Gresho, S.T. Chang, R.L. Lee, C.D. Upson, Int. J. Numer. Methods in Fluids 4 (1984) 557-598.

[72] J.F. Thompson, Z.U.A. Warsi, C. Wayne Mastin, Numerical Grid Generation - Foundations and Applications, Elsevier, Amsterdam, 1985.

[73] M. Yerushalmi, H. Pfeffer, R. Shinnar, J. Fluid Mech. 38 (1969) 689. 